\title{
Poaceae em uma planície de inundação no Brasil: distribuição espacial e conservação ${ }^{1}$
}

Poaceae in a Brazilian floodplain: spatial distribution and conservation

\author{
Kazue Kawakita ${ }^{2,6}$, Alan Charles Fontana ${ }^{3}$, Jéssica Magon Garcia ${ }^{4}$, Rodrigo S. Rodrigues ${ }^{5}$ \\ \& Tarciso S. Filgueiras ${ }^{5}$
}

\begin{abstract}
Resumo
Com 94 espécies, Poaceae é uma das maiores famílias botânicas ocorrentes na Planície de Inundação do Alto Rio Paraná (PIAP) e seu entorno, localizada a $22^{\circ} 38^{\prime}-22^{\circ} 57^{\prime} \mathrm{S}$ e $53^{\circ} 5^{\prime}-53^{\circ} 36^{\prime} \mathrm{O}$, a qual abrange áreas dos estados do Mato Grosso do Sul e do Paraná, Brasil. Sazonalmente inundável, a PIAP faz parte da Área de Proteção Ambiental Federal das Ilhas e Várzeas do Rio Paraná (APA-IVRP) e do Parque Estadual das Várzeas do Rio Ivinhema (PEVRI). Com o objetivo de ampliar os conhecimentos botânicos, ecológicos e oferecer subsídios que possam contribuir para a conservação, no presente trabalho foi avaliado o padrão de distribuição espacial das gramíneas na PIAP e no seu entorno. Para as espécies ocorrentes na PIAP foram considerados: fitofisionomia, exposição à luz, via fotossintética, condição hídrica do substrato em que preferencialmente ocorrem, classificação da morfovegetação, frequências absoluta e relativa, origem e Domínio Fitogeográfico. Os resultados mostram que as espécies distribuem-se espacialmente de acordo com a configuração geomorfológica da PIAP, aqui representadas em dois perfis esquemáticos.
\end{abstract}

Palavras-chave: áreas úmidas, brejo, Gramineae, Rio Paraná, vegetação ripária.

\begin{abstract}
With 94 species, Poaceae is one of the largest botanical families of the Upper Paraná River Floodplain (PIAP) and its environs, located between $22^{\circ} 38^{\prime}-22^{\circ} 57^{\prime} \mathrm{S}$ and $53^{\circ} 5^{\prime}-53^{\circ} 36^{\prime} \mathrm{W}$, and covering areas of the Brazilian states of Mato Grosso do Sul and Paraná. Seasonally flooded, PIAP is part of the The Environmental Protection Area of the Islands and floodplains of the Paraná River (APA-IVRP) and State Park of the Ivinhema River Floodplain (PEVRI). Aiming to improve the botanical and ecological knowledge of the area and therefore contribute to its conservation, we studied the distribution pattern of the grasses of PIAP and its environs. For the grasses of PIAP we considered: phytophysiognomy, light exposure, photosynthetic pathway, water condition of the substrate where they preferably occur, classification of the morphovegetation, and the absolute and relative frequencies. The results show that the species are distributed according to the geomorphological configuration of the PIAP, which are presented here in two schematic profiles.
\end{abstract}

Key words: wetlands, swamp, Gramineae, Paraná River, riparian vegetation.

\section{Introdução}

Compondo cerca de $40 \%$ da cobertura vegetal no mundo, as Poaceae estão entre as angiospermas mais amplamente distribuídas, principalmente nas regiões tropicais e subtropicais (Watson \& Dallwitz 1992). Com 1.479 espécies registradas para o Brasil, distribuem-se por todos os biomas e estados (Filgueiras et al. 2016). Ocorrem nos mais variados tipos de ambientes, tanto campestres quanto florestais (Oliveira \& Longhi-Wagner 2005; Boldrini et al. 2008; Schmidt \& Longhi-Wagner 2009).

Material disponível também em <https://doi.org/10.6084/m9.figshare.6146516.v1>

\footnotetext{
${ }^{1}$ Parte da tese de doutorado da primeira autora.

${ }^{2}$ Universidade Estadual de Maringá - Nupélia/UEM, Av. Colombo 5790, B1. G-80, 87020-900, Maringá, PR, Brasil.

${ }^{3}$ Serviço Social da Indústria (SESI), Av. Aviação 1851, 86812-470, Apucarana, PR, Brasil.

${ }^{4}$ Universidade Estadual de Londrina, Prog. Pós-graduação em Ciências Biológicas, Rod. Celso Garcia Cid, PR-445, km 380, Campus Universitário, 86057-970, Londrina, PR, Brasil.

${ }^{5}$ Instituto de Botânica, Prog. Pós-graduação em Biodiversidade Vegetal e Meio Ambiente, Núcleo de Pesquisa Curadoria do Herbário SP, Av. Miguel Stéfano 3687, 04301-902, São Paulo, SP, Brasil.

${ }^{6}$ Autor para correspondência: kazue@nupelia.uem.br
} 
Poaceae representa a segunda maior família fanerogâmica da Planície de Inundação do Alto Rio Paraná (PIAP) (Souza et al. 1997, 2009). São registradas 94 espécies de gramíneas nesta planície (Kawakita et al. 2016), cujos indivíduos encontram-se distribuídos, principalmente, nos campos naturais, no entorno de lagoas (Souza et al. 2004b) ou em ambientes antropizados, como pastagens ativas ou abandonadas.

A PIAP encontra-se condicionada a ciclos hidrológicos sazonais e plurianuais, além de uma série de fatores impactantes como: presença de barragens hidrelétricas a montante, exploração agropecuária, fogo, drenagem, extração de argila e presença de espécies exóticas invasoras (Campos \& Souza 1997; Hayakawa et al. 2010; UEM 2017). A dinâmica fluvial originada pela inundação proporciona uma complexidade de hábitats e distintos tipos de cobertura vegetal, constituindose a principal forma de realce das formas de relevo existentes na região (Rocha \& Comunello 2009). A relação entre a geomorfologia e a distribuição da vegetação tem sido demonstrada em diferentes perfis fitotopográficos da PIAP (Fachini 2001; Corradini et al. 2006, 2008; Stevaux et al. 2013).

Campos \& Souza (1997) e Campos (2004) estudaram a distribuição espacial da vegetação da PIAP, com suas formações florestais, não florestais, além das áreas antrópicas, relacionadas aos fatores hidrodinâmicos e hidrossedimentológicos. A cobertura florestal original da região encontrase reduzida a pequenos fragmentos florestais, principalmente nas áreas próximas ao leito do Rio Paraná e no conjunto de ilhas que formam o arquipélago do Rio Paraná (Campos \& Souza 1997).

Com relação às Poaceae, ainda não haviam sido realizados estudos com enfoque sobre a distribuição espacial das espécies na PIAP. Objetivando preencher esta lacuna e ampliar o conhecimento botânico e ecológico acerca das Poaceae na PIAP e seu entorno, foi realizada uma análise do padrão de distribuição espacial das espécies nesta planície, além de destacar alguns aspectos sobre conservação.

\section{Material e Métodos}

A Planície de Inundação do Alto Rio Paraná (PIAP) e seu entorno localizam-se entre $22^{\circ} 38^{\prime}-$ $22^{\circ} 57^{\prime} \mathrm{S}$ e $53^{\circ} 5^{\prime}-53^{\circ} 36^{\prime} \mathrm{O}$, na divisa entre os estados do Mato Grosso do Sul e do Paraná. Esta planície compreende parte do Alto Rio Paraná, que se estende desde a confluência entre o Rio Paranaíba e o Rio Grande até os antigos Saltos de Sete Quedas em Guaíra, PR (Souza Filho 1994; Souza Filho \& Stevaux 2004). A área de estudo abrange o Rio Paraná, sua porção oriental (margem esquerda) que inclui os municípios paranaenses (Marilena, São Pedro do Paraná, Porto Rico e Querência do Norte) e ocidental (margem direita) sul-matogrossenses (Batayporã, Ivinhema, Taquarussu e Jateí).

Sazonalmente inundável, a PIAP faz parte da APA-IVRP (Área de Proteção Ambiental Federal das Ilhas e Várzeas do Rio Paraná) e do PEVRI (Parque Estadual das Várzeas do Rio Ivinhema). Inserida na metade superior da APA-IVRP, a PIAP abrange cerca de $1.100 \mathrm{~km}^{2}$ (526.752 ha), constitui o Sítio 6 do Programa Nacional de Pesquisas Ecológicas de Longa Duração (PELD), e faz parte da Reserva da Biosfera da Mata Atlântica - MAB/ UNESCO (Agostinho et al. 2004; UEM 2016).

O relevo da região é marcado por uma clara assimetria entre as duas margens do Rio Paraná. $\mathrm{Na}$ margem esquerda (paranaense) o rio apresenta relevo ondulado com paredões de Arenito da Formação Caiuá, formando barrancas de até 10-20 m de altura (geralmente interflúvios dos afluentes), além de estreitas faixas inundáveis, com cotas de 280-400 m. Na margem direita (sul-matogrossense), apresenta relevo suave com uma expressiva planície de inundação, também denominada várzea (Souza Filho 1994; Stevaux \& Souza-Filho 1997; Souza-Filho \& Stevaux 2004). As partes mais elevadas são representadas por diques marginais e estão situadas entre 238-242 m de altitude. As partes mais baixas, representadas pelos canais ativos, canais abandonados e pelas bacias de inundação, situam-se entre 232-237 m de altitude (Fragal et al. 2012).

O clima da região é tropical-subquente, com temperaturas médias anuais de $20^{\circ} \mathrm{C}$ e precipitações superiores a $1.500 \mathrm{~mm} / \mathrm{ano}$ (IBGE 2012).

Sob o domínio da Mata Atlântica, entre o Cerrado sul-matogrossense e a Floresta Estacional Semidecidual paranaense, a PIAP apresenta como elementos típicos as formações de várzea e florestas ripárias (Souza et al. 1997; IBGE 2012).

Nas ilhas do Rio Paraná, a vegetação enquadrase nas formações pioneiras de influência fluvial, com suas diversas associações (Campos \& Souza 1997). Em áreas topograficamente rebaixadas e sazonalmente alagáveis ocorrem campos naturais, principalmente em áreas do PEVRI (Kawakita et al. 2016).

A partir da listagem das Poaceae da PIAP (Kawakita et al. 2016), de dados da literatura, dos rótulos das exsicatas e de observações em campo, 
obtidos no período de 2010-2015, foi elaborada uma tabela comparativa com informações específicas para cada táxon. Constam ainda nesta tabela os valores de frequências absoluta (FA) e relativa (FR), ambos em porcentagem. O cálculo de FA foi obtido a partir da relação entre o número de locais de amostragem de cada espécie e o número total de espécies amostradas. O cálculo de FR foi obtido segundo Braun-Blanquet (1979), relacionando-se FA de cada espécie e a somatória de FA de todas as espécies.

A distribuição e a ocorrência nos diferentes domínios fitogeográficos seguem Filgueiras et al. (2017). A classificação em grupos de morfovegetação é apresentada de acordo com Stevaux et al. (2013) (Anl = Tree-dominated natural levee [diques marginais com predominância de árvores]; SHufp = Shrubby upper floodplain [várzea alta com arbustos]; SHmfp = Shrubbyherbaceous mid-floodplain [várzea média com arbustos e herbáceas]; GHlfp = Grass-herbaceous lower floodplain [várzea baixa com gramíneas e herbáceas] e respectivos ambientes).

Informações referentes às vias fotossintéticas foram retiradas da literatura (Tab. 1).

Para avaliação da distribuição espacial, considerou-se o Rio Paraná e a planície de inundação como uma unidade ecológica, formando o sistema rio-planície de inundação (Junk et al. 1989). Tendo em vista a assimetria do relevo entre as margens do Rio Paraná e as inúmeras ilhas, as espécies de gramíneas da PIAP foram classificadas em três sub-sistemas: Sub-sistema Rio Paraná Margem Esquerda (SRPME) (córrego Areia Branca, ribeirão São Pedro, córrego Caracu, Rio Paraná margem esquerda), Sub-sistema Rio Paraná Central Ilhas e Barras (SRPCI) (arquipélago do Rio Paraná) e Sub-sistema Rio Paraná Margem Direita (SRPMD) (Rio Baía, Rio Samambaia, córrego Baile, canal do Rio Baía, canal Corutuba, Rio Ivinhema, Ribeirão Vitória, Rio Guiraí, Rio Curupaí, Rio Paraná margem direita), conforme as localidades de amostragem (Souza et al. 2004a).

Para melhor visualização da distribuição espacial das espécies na PIAP e do seu entorno não inundável, confeccionou-se um perfil graminosofisionômico, demonstrando os diferentes ambientes com base nos perfis fitotopográficos descritos por Fachini (2001), Corradini (2006) e Stevaux et al. (2013). Foram selecionadas e incluídas no perfil esquemático sete espécies de gramíneas representativas de cada um dos diferentes ambientes desta planície.
A indicação do Status das espécies, se nativas ou naturalizadas, foi obtida a partir de consulta à base de dados The Brazil Flora Group (BFG 2015). Também foi avaliado se, dentre as naturalizadas, alguma é considerada invasora na literatura e em observações a campo. As invasoras podem ser consideradas como indicadores biológicos do grau de perturbação ambiental a que uma determinada área está submetida (Filgueiras 1990; Shirasuna et al. 2013).

\section{Resultados e Discussão}

Com 94 espécies, Poaceae é uma das maiores famílias botânicas ocorrentes na Planície de Inundação do Alto Rio Paraná (PIAP) e entorno (Kawakita et al. 2016). Essas espécies ocorrem também em um ou mais biomas, sendo: Mata Atlântica (88 spp. ou 93,62\%), Cerrado (85 spp. ou 90,42\%), Amazônia (72 spp. ou 76,59\%), Caatinga (55 spp. ou 58,51\%), Pantanal (47 spp. ou 50\%) e Pampa (32 spp. ou 34,04\%) (Tab. 1). Destacaramse com maiores valores percentuais de número de espécies a Mata Atlântica e o Cerrado. Este fato está em conformidade com o esperado, uma vez que a área amostrada abrange a zona de transição entre os dois domínios.

As espécies ocorrem nos diferentes ambientes da planície, no entorno de córregos, rios, riachos, lagoas, etc., nas diversas formações vegetais, desde os campos naturais aos antropizados, como nas pastagens ativas ou abandonadas. Ocorrem também nos fragmentos florestais secundários, nas áreas mais abertas, como nas capoeiras e áreas de reflorestamento, inclusive nos fragmentos florestais mais preservados. Do total de espécies registradas, $71(75,53 \%)$ foram amostradas em áreas abertas (campos naturais e pastagens ativas ou abandonadas em diferentes estágios sucessionais), 23 (24,47\%) foram amostradas em ambientes florestais (floresta secundária, mata ciliar, borda seca de mata ciliar e fragmento de Floresta Estacional Semidecidual, vegetação em sucessão natural de área aberta para floresta, margem sombreada e alagada de ilhas e de florestas secundárias) (Tab. 1).

Das 30 lagoas na PIAP avaliadas por Souza $e t$ al. (2004a) (incluindo aquelas localizadas nas ilhas do Rio Paraná e seus tributários, principalmente Rio Baía e Rio Ivinhema), nove (30\%) apresentam dominância fisionômica graminosa, composta, principalmente, por espécies nativas, tais como Coleataenia prionitis, Hymenachne pernambucensis e Panicum tricholaenoides. 
Tabela 1 - Lista das espécies de Poaceae da Planície de Inundação do Alto Rio Paraná e seu entorno, Mato Grosso do Sul e Paraná, Brasil. Abreviações: FF = Fitofisionomia $(F=$ florestal; $A=$ aberta); EAL = Exposição à Luz $(E=$ esciófila; $H=$ heliófila; $\mathrm{U}=$ umbrófila); $\mathrm{VF}=$ via fotossintética; $\mathrm{CHS}=$ condição hídrica do substrato ( $\mathrm{DRE}=$ drenado; $\mathrm{BDR}=$ bem drenado; $\mathrm{SAT}=$ saturado; SED = sedimento [lama]); UMV = unidades de morfovegetação, segundo Stevaux et al. $(2013)($ Anl $=$ Tree-dominated natural levee; SHufp = Shrubby upper floodplain; SHmfp = Shrubby-herbaceous mid-floodplain; GHlfp = Grass-herbaceous lower floodplain e respectivos ambientes); FA = frequência absoluta; FR = frequência relativa. Status: NT = Nativa; $\mathrm{NZ}=$ Naturalizada [exótica]. Domínio fitogeográfico: $\mathrm{AM}=$ Amazônia; $\mathrm{CA}=$ Caatinga; $\mathrm{CE}=\mathrm{Cerrado} ; \mathrm{MA}=$ Mata Atlântica; PP = Pampa; PT = Pantanal.

Table 1 - List of the species of Poaceae from the Upper Paraná River Floodplain and its surroundings, Mato Grosso do Sul and Paraná, Brazil. Acronyms: $\mathrm{FF}=$ Phytophysiognomy ( $\mathrm{F}=$ forest; $\mathrm{A}=$ open formation); $\mathrm{EAL}=$ Light Exposure $(\mathrm{E}=$ esciophylous; $\mathrm{H}=$ light-demanding; $\mathrm{U}=$ shade-tolerant); $\mathrm{VF}=$ photosynthetic pathway; $\mathrm{CHS}=$ water condition of the substrate $(\mathrm{DRE}=$ drained; $\mathrm{BDR}=$ well drained; $\mathrm{SAT}=$ saturated; $\mathrm{SED}$ = sediment [mud]); UMV = morphovegetation units, according to Stevaux et al. (2013) (Anl=Tree-dominated natural levee; SHufp $=$ Shrubby upperfloodplain; SHmfp = Shrubby-herbaceous mid-floodplain; GHlfp = Grass-herbaceous lower floodplain and respective environments); FA = absolute frequence; FR = relative frequence. Status: NT = native; NZ = naturalized [exotic]; Phytogeographic domain (AM = Amazon reinforest; $\mathrm{CA}=$ Caatinga; $\mathrm{CE}=$ Central Brazilian savanna; $\mathrm{MA}=$ Atlantic rainforest $\mathrm{PP}=$ Brazilian Pampa; $\mathrm{PT}=$ Pantanal).

\begin{tabular}{|c|c|c|c|c|c|c|c|c|c|c|c|c|c|c|}
\hline \multirow{2}{*}{ Espécie } & \multirow{2}{*}{ FF } & \multirow{2}{*}{ EAL } & \multirow{2}{*}{ VF } & \multirow{2}{*}{ CHS } & \multirow{2}{*}{ UMV } & \multirow{2}{*}{$\begin{array}{l}\text { FA } \\
(\%)\end{array}$} & \multirow{2}{*}{$\begin{array}{l}\text { FR } \\
(\%)\end{array}$} & \multirow{2}{*}{ Status } & \multicolumn{6}{|c|}{ Domínio Fitogeográfico } \\
\hline & & & & & & & & & $\mathbf{A M}$ & $\mathbf{C A}$ & $\mathbf{C E}$ & MA & $\mathbf{P P}$ & PT \\
\hline $\begin{array}{l}\text { Acroceras zizanioides (Kunth) } \\
\text { Dandy* }\end{array}$ & $\mathrm{F}$ & $\mathrm{U}$ & $\mathrm{C}^{9}$ & DRE & $\begin{array}{l}\text { Anl, } \\
\text { SHmfp }\end{array}$ & 42 & 2,55 & NT & $\mathrm{X}$ & $\mathrm{X}$ & $\mathrm{X}$ & $\mathrm{X}$ & & \\
\hline Andropogon bicornis L. & A & $\mathrm{H}$ & $\mathrm{C}^{9}{ }^{9}$ & $\begin{array}{l}\text { DRE, } \\
\text { BDR }\end{array}$ & $\begin{array}{l}\text { Anl, } \\
\text { SHfp }\end{array}$ & 23,81 & 1,45 & NT & $\mathrm{X}$ & $\mathrm{X}$ & $\mathrm{X}$ & $\mathrm{X}$ & $\mathrm{X}$ & $\mathrm{X}$ \\
\hline $\begin{array}{l}\text { Andropogon hypogynus } \\
\text { Hack.* }\end{array}$ & A & $\mathrm{H}$ & $\mathrm{C} 4^{12}$ & DRE & SHfp & 14,28 & 0,87 & NT & & & $\mathrm{X}$ & & & $X$ \\
\hline Arundo donax $\mathrm{L}$. & A & $\mathrm{H}$ & $\mathrm{C} 3^{2}$ & DRE & - & 4,46 & 0,29 & NZ & & & $\mathrm{X}$ & $\mathrm{X}$ & & \\
\hline $\begin{array}{l}\text { Axonopus fissifolius (Raddi) } \\
\text { Kuhlm.* }\end{array}$ & A & $\mathrm{H}$ & $\mathrm{C}^{7,9}$ & $\begin{array}{l}\text { DRE, } \\
\text { SAT }\end{array}$ & $\begin{array}{l}\text { Anl, } \\
\text { GHlfp }\end{array}$ & 28,57 & 1,73 & NT & $\mathrm{X}$ & $\mathrm{X}$ & $\mathrm{X}$ & $\mathrm{X}$ & & \\
\hline $\begin{array}{l}\text { Axonopus leptostachyus } \\
\text { (Flüggé) Hitchc.* }\end{array}$ & A & $\mathrm{H}$ & $\mathrm{C} 4^{12}$ & DRE & - & 9,52 & 0,58 & NT & $\mathrm{X}$ & & $\mathrm{X}$ & $\mathrm{X}$ & & \\
\hline $\begin{array}{l}\text { Axonopus suffultus } \\
\text { (Mikan ex Trin.) Parodi* }\end{array}$ & A & $\mathrm{H}$ & $\mathrm{C} 4^{12}$ & DRE & - & 4,76 & 0,29 & NT & $\mathrm{X}$ & & $\mathrm{X}$ & $\mathrm{X}$ & & \\
\hline Bambusa tuldoides Munro & $\mathrm{F}$ & $\mathrm{U}$ & $\mathrm{C} 3^{12}$ & $\begin{array}{l}\text { BDR, } \\
\text { DRE }\end{array}$ & - & 19 & 1,15 & NZ & & & $\mathrm{X}$ & $\mathrm{X}$ & & \\
\hline $\begin{array}{l}\text { Bambusa vulgaris Schrad. ex } \\
\text { J.C. Wendl. }\end{array}$ & $\mathrm{F}$ & $\mathrm{U}$ & $\mathrm{C} 3^{12}$ & $\begin{array}{l}\text { BDR, } \\
\text { DRE }\end{array}$ & - & 9,52 & 0,58 & $\mathrm{NZ}$ & $\mathrm{X}$ & $\mathrm{X}$ & $\mathrm{X}$ & $\mathrm{X}$ & & $X$ \\
\hline $\begin{array}{l}\text { Cenchrus americanus (L.) } \\
\text { Morrone }\end{array}$ & A & $\mathrm{H}$ & $\mathrm{C} 4^{12}$ & DRE & $A n l$ & 4,76 & 0,29 & NZ & & & $\mathrm{X}$ & $\mathrm{X}$ & & \\
\hline Cenchrus echinatus L. & A & $\mathrm{H}$ & $\mathrm{C} 4^{12}$ & DRE & - & 14,28 & 0,87 & NT & $\mathrm{X}$ & $\mathrm{X}$ & $\mathrm{X}$ & $\mathrm{X}$ & & $X$ \\
\hline $\begin{array}{l}\text { Cenchrus purpureus } \\
\text { (Schumach.) Morrone }\end{array}$ & A & $\mathrm{H}$ & $\mathrm{C} 4^{12}$ & DRE & - & 23,8 & 1,44 & $\mathrm{NZ}$ & $\mathrm{X}$ & $\mathrm{X}$ & $\mathrm{X}$ & $X$ & & \\
\hline Chloris elata Desv. & A & $\mathrm{H}$ & $\mathrm{C} 4^{12}$ & BDR & - & 4,76 & 0,29 & NT & $\mathrm{X}$ & $\mathrm{X}$ & $\mathrm{X}$ & $\mathrm{X}$ & $\mathrm{X}$ & $X$ \\
\hline $\begin{array}{l}\text { Chusquea ramosissima } \\
\text { Lindm. }\end{array}$ & $\mathrm{F}$ & $\mathrm{E}$ & $\mathrm{C} 3^{12}$ & $\begin{array}{l}\mathrm{BDR}, \\
\mathrm{DRE}\end{array}$ & - & 9,52 & 0,5 & NT & & & $\mathrm{X}$ & $\mathrm{X}$ & & \\
\hline $\begin{array}{l}\text { Coleataenia prionitis (Nees) } \\
\text { Soreng }\end{array}$ & A & $\mathrm{H}$ & $\begin{array}{l}\mathrm{C} 3 / \\
\mathrm{C} 4^{11}\end{array}$ & $\begin{array}{l}\text { DRE, } \\
\text { SAT }\end{array}$ & $\begin{array}{l}\text { Anl, } \\
\text { GHlfp }\end{array}$ & 28,57 & 1,73 & NT & & & $\mathrm{X}$ & & & \\
\hline
\end{tabular}




\begin{tabular}{|c|c|c|c|c|c|c|c|c|c|c|c|c|c|c|}
\hline \multirow{2}{*}{ Espécie } & \multirow{2}{*}{ FF } & \multirow{2}{*}{ EAL } & \multirow{2}{*}{$\mathbf{V F}$} & \multirow{2}{*}{ CHS } & \multirow{2}{*}{ UMV } & \multirow{2}{*}{$\begin{array}{l}\text { FA } \\
(\%)\end{array}$} & \multirow{2}{*}{$\begin{array}{l}\text { FR } \\
(\%)\end{array}$} & \multirow{2}{*}{ Status } & \multicolumn{6}{|c|}{ Domínio Fitogeográfico } \\
\hline & & & & & & & & & $\mathbf{A M}$ & CA & $\mathbf{C E}$ & MA & PP & PT \\
\hline $\begin{array}{l}\text { Cymbopogon nardus (L.) } \\
\text { Rendle }\end{array}$ & A & $\mathrm{H}$ & $\mathrm{C} 4^{12}$ & $\mathrm{BDR}$ & - & 4,76 & 0,29 & $\mathrm{NZ}$ & & & $\mathrm{X}$ & $\mathrm{X}$ & & \\
\hline Cynodon dactylon (L.) Pers.* & A & $\mathrm{H}$ & $\mathrm{C} 4^{9}$ & $\begin{array}{l}\text { BDR, } \\
\text { DRE }\end{array}$ & SHufp & 38,09 & 2,31 & NT & $\mathrm{X}$ & $\mathrm{X}$ & $\mathrm{X}$ & $\mathrm{X}$ & $\mathrm{X}$ & $\mathrm{X}$ \\
\hline Cynodon nlemfuensis Vand. & A & $\mathrm{H}$ & $\mathrm{C} 4^{12}$ & $\mathrm{BDR}$ & - & 4,76 & 0,29 & $\mathrm{NZ}$ & & $\mathrm{X}$ & $\mathrm{X}$ & $\mathrm{X}$ & $\mathrm{X}$ & $\mathrm{X}$ \\
\hline $\begin{array}{l}\text { Dendrocalamus asper } \\
\text { (Schult. \& Schult. f.) Baker ex } \\
\text { K. Heyne }\end{array}$ & $\mathrm{F}$ & $\mathrm{U}$ & $\mathrm{C} 3^{12}$ & BDR & - & 4,76 & 0,29 & $\mathrm{NZ}$ & & & $\mathrm{X}$ & $\mathrm{X}$ & & \\
\hline $\begin{array}{l}\text { Dichanthelium surrectum } \\
\text { (Chase ex Zuloaga \& } \\
\text { Morrone) Zuloaga* }\end{array}$ & $\mathrm{F}$ & $\mathrm{H}$ & $\mathrm{C} 3^{1}$ & $\begin{array}{l}\text { DRE, } \\
\text { SED }\end{array}$ & GHlfp & 9,52 & 0,58 & NT & & & $\mathrm{X}$ & $\mathrm{X}$ & & \\
\hline $\begin{array}{l}\text { Digitaria ciliaris (Retz.) } \\
\text { Koeler }\end{array}$ & A & $\mathrm{H}$ & $\mathrm{C} 4^{9}$ & BDR & - & 14,28 & 0,87 & $\mathrm{NZ}$ & $\mathrm{X}$ & $\mathrm{X}$ & $\mathrm{X}$ & $\mathrm{X}$ & $\mathrm{X}$ & $\mathrm{X}$ \\
\hline Digitaria insularis (L.) Fedde & A & $\mathrm{H}$ & $\mathrm{C} 4^{9}$ & $\begin{array}{l}\text { BDR, } \\
\text { DRE }\end{array}$ & - & 14,28 & 0,87 & $\mathrm{NZ}$ & $\mathrm{X}$ & $\mathrm{X}$ & $\mathrm{X}$ & $\mathrm{X}$ & $\mathrm{X}$ & $\mathrm{X}$ \\
\hline Digitaria violascens Link & A & $\mathrm{H}$ & $\mathrm{C} 4^{9}$ & $\begin{array}{l}\text { BDR, } \\
\text { DRE }\end{array}$ & - & 9,52 & 0,58 & $\mathrm{NZ}$ & $\mathrm{X}$ & $\mathrm{X}$ & $\mathrm{X}$ & $\mathrm{X}$ & $\mathrm{X}$ & $\mathrm{X}$ \\
\hline $\begin{array}{l}\text { Echinochloa crus-galli (L.) P. } \\
\text { Beauv.* }\end{array}$ & A & $\mathrm{H}$ & $\mathrm{C} 4^{12}$ & $\begin{array}{l}\text { DRE, } \\
\text { SAT }\end{array}$ & - & 19 & 1,15 & $\mathrm{NZ}$ & $\mathrm{X}$ & $\mathrm{X}$ & $\mathrm{X}$ & $\mathrm{X}$ & $\mathrm{X}$ & $\mathrm{X}$ \\
\hline $\begin{array}{l}\text { Echinochloa crus-pavonis } \\
\text { (Kunth) Schult.* }\end{array}$ & A & $\mathrm{H}$ & $\mathrm{C} 4^{9}$ & $\begin{array}{l}\text { DRE, } \\
\text { SAT }\end{array}$ & - & 9,52 & 0,58 & $\mathrm{NZ}$ & $\mathrm{X}$ & $\mathrm{X}$ & $\mathrm{X}$ & $\mathrm{X}$ & $\mathrm{X}$ & $\mathrm{X}$ \\
\hline Eleusine indica (L.) Gaertn. & A & $\mathrm{H}$ & $\mathrm{C} 4^{9}$ & $\mathrm{BDR}$ & - & 19 & 1,15 & $\mathrm{NZ}$ & $\mathrm{X}$ & $\mathrm{X}$ & $\mathrm{X}$ & $\mathrm{X}$ & $\mathrm{X}$ & $\mathrm{X}$ \\
\hline $\begin{array}{l}\text { Eragrostis articulata } \\
\text { (Schrank) Nees }\end{array}$ & A & $\mathrm{H}$ & $\mathrm{C} 4^{12}$ & $\begin{array}{l}\text { BDR, } \\
\text { DRE }\end{array}$ & - & 14,28 & 0,87 & NT & $\mathrm{X}$ & $\mathrm{X}$ & $\mathrm{X}$ & $\mathrm{X}$ & & \\
\hline $\begin{array}{l}\text { Eragrostis bahiensis Schrad. } \\
\text { ex Schult.* }\end{array}$ & A & $\mathrm{H}$ & $\mathrm{C} 4^{9}$ & $\begin{array}{l}\text { BDR, } \\
\text { DRE }\end{array}$ & - & 33,33 & 2,02 & $\mathrm{NZ}$ & $\mathrm{X}$ & $\mathrm{X}$ & $\mathrm{X}$ & $\mathrm{X}$ & $\mathrm{X}$ & \\
\hline Eragrostis ciliaris (L.) R. Br. & A & $\mathrm{H}$ & $\mathrm{C} 4^{9}$ & $\begin{array}{l}\text { BDR, } \\
\text { DRE }\end{array}$ & - & 19 & 1,15 & NT & $\mathrm{X}$ & $\mathrm{X}$ & $\mathrm{X}$ & $\mathrm{X}$ & & $\mathrm{X}$ \\
\hline $\begin{array}{l}\text { Eragrostis hypnoides (Lam.) } \\
\text { Britton }\end{array}$ & A & $\mathrm{H}$ & $\mathrm{C} 4^{9}$ & DRE & - & 9,52 & 0,58 & NT & $\mathrm{X}$ & $\mathrm{X}$ & $\mathrm{X}$ & $\mathrm{X}$ & $\mathrm{X}$ & \\
\hline $\begin{array}{l}\text { Eragrostis pilosa (L.) P. } \\
\text { Beauv. }\end{array}$ & A & $\mathrm{H}$ & $\mathrm{C} 4^{9}$ & BDR & - & 9,52 & 0,58 & $\mathrm{NZ}$ & $\mathrm{X}$ & $\mathrm{X}$ & $\mathrm{X}$ & $\mathrm{X}$ & & \\
\hline $\begin{array}{l}\text { Eragrostis tenella (L.) P. } \\
\text { Beauv. ex Roem. \& Schult. }\end{array}$ & A & $\mathrm{H}$ & $\mathrm{C} 4^{9}$ & BDR & - & 4,76 & 0,29 & $\mathrm{NZ}$ & $\mathrm{X}$ & $\mathrm{X}$ & $\mathrm{X}$ & $\mathrm{X}$ & & $\mathrm{X}$ \\
\hline Eriochloa polystachya Kunth & A & $\mathrm{H}$ & $\mathrm{C} 4^{9}$ & SAT & - & 4,76 & 0,29 & $\mathrm{NZ}$ & $\mathrm{X}$ & & & $\mathrm{X}$ & & \\
\hline $\begin{array}{l}\text { Eriochloa punctata (L.) Desv. } \\
\text { ex Ham.* }\end{array}$ & A & $\mathrm{H}$ & $\mathrm{C} 4^{12}$ & $\begin{array}{l}\text { BDR, } \\
\text { DRE }\end{array}$ & - & 23,8 & 1,44 & NT & $X$ & $\mathrm{X}$ & $X$ & $X$ & & \\
\hline $\begin{array}{l}\text { Guadua chacoensis (Rojas) } \\
\text { Londoño \& P.M. Peterson* }\end{array}$ & $\mathrm{F}$ & $\mathrm{U}$ & $\mathrm{C} 3^{12}$ & DRE & $A n l$ & 23,8 & 1,44 & NT & & & & $X$ & & $X$ \\
\hline
\end{tabular}




\begin{tabular}{|c|c|c|c|c|c|c|c|c|c|c|c|c|c|c|}
\hline \multirow{2}{*}{ Espécie } & \multirow{2}{*}{ FF } & \multirow{2}{*}{ EAL } & \multirow{2}{*}{ VF } & \multirow{2}{*}{ CHS } & \multirow{2}{*}{ UMV } & \multirow{2}{*}{$\begin{array}{c}\text { FA } \\
(\%)\end{array}$} & \multirow{2}{*}{$\begin{array}{l}\text { FR } \\
(\%)\end{array}$} & \multirow{2}{*}{ Status } & \multicolumn{6}{|c|}{ Domínio Fitogeográfico } \\
\hline & & & & & & & & & $\mathbf{A M}$ & $\mathbf{C A}$ & CE & MA & PP & PT \\
\hline Guadua paniculata Munro* & $\mathrm{F}$ & $\mathrm{U}$ & $\mathrm{C} 3^{12}$ & DRE & $A n l$ & 47,62 & 2,89 & NT & $\mathrm{X}$ & & $\mathrm{X}$ & $\mathrm{X}$ & & \\
\hline Guadua sp. & $\mathrm{F}$ & $\mathrm{U}$ & $\mathrm{C} 3^{12}$ & DRE & Anl & 9,52 & 0,58 & NT & - & - & - & - & - & - \\
\hline $\begin{array}{l}\text { Gynerium sagittatum (Aubl.) } \\
\text { P. Beauv. }\end{array}$ & $\mathrm{F}$ & $\mathrm{H}$ & $\mathrm{C} 3^{9}$ & $\begin{array}{l}\text { BDR, } \\
\text { DRE }\end{array}$ & Anl & 14,28 & 0,87 & NT & $\mathrm{X}$ & & $X$ & $X$ & & $\mathrm{X}$ \\
\hline $\begin{array}{l}\text { Hemarthria altissima (Poir.) } \\
\text { Stapf \& C.E. Hubb. }\end{array}$ & $\mathrm{A}$ & $\mathrm{H}$ & $\mathrm{C} 4^{12}$ & $\begin{array}{l}\text { DRE, } \\
\text { SAT }\end{array}$ & - & 4,76 & 0,29 & NT & $\mathrm{X}$ & & & $\mathrm{X}$ & $\mathrm{X}$ & $\mathrm{X}$ \\
\hline $\begin{array}{l}\text { Hildaea pallens (Sw.) C. Silva } \\
\text { \& R.P. Oliveira }\end{array}$ & $\mathrm{F}$ & $\mathrm{U}$ & $\mathrm{C} 3^{9}$ & SAT & - & 9,52 & 0,58 & NT & $\mathrm{X}$ & $\mathrm{X}$ & $\mathrm{X}$ & $\mathrm{X}$ & & \\
\hline $\begin{array}{l}\text { Hymenachne amplexicaulis } \\
\text { (Rudge) Nees* }\end{array}$ & $\mathrm{F}$ & $\mathrm{E}$ & $\mathrm{C} 3^{9}$ & $\begin{array}{l}\text { SAT, } \\
\text { SED }\end{array}$ & GHlfp & 23,8 & 1,44 & NT & $\mathrm{X}$ & $\mathrm{X}$ & $\mathrm{X}$ & $\mathrm{X}$ & & $\mathrm{X}$ \\
\hline $\begin{array}{l}\text { Hymenachne donacifolia } \\
\text { (Raddi) Chase }\end{array}$ & A & $\mathrm{H}$ & $\begin{array}{l}\mathrm{C} 3 / \\
\mathrm{C} 4^{11}\end{array}$ & SAT & GHlfp & 14,28 & 0,87 & NT & $\mathrm{X}$ & $\mathrm{X}$ & $\mathrm{X}$ & $X$ & & \\
\hline $\begin{array}{l}\text { Hymenachne pernambucensis } \\
\text { (Spreng.) Zuloaga* }\end{array}$ & A & $\mathrm{H}$ & $\begin{array}{l}\mathrm{C} 3 / \\
\mathrm{C} 4^{11}\end{array}$ & $\begin{array}{l}\text { DRE, } \\
\text { SAT }\end{array}$ & $A n l$ & 61,9 & 3,76 & NT & $\mathrm{X}$ & $X$ & $\mathrm{X}$ & $X$ & & \\
\hline $\begin{array}{l}\text { Lasiacis ligulata Hitchc. \& } \\
\text { Chase }\end{array}$ & $\mathrm{F}$ & $\mathrm{E}$ & $\mathrm{C} 3^{12}$ & DRE & - & 14,28 & 0,87 & NT & $\mathrm{X}$ & $\mathrm{X}$ & $\mathrm{X}$ & $\mathrm{X}$ & & \\
\hline Leersia hexandra Sw.* & $\mathrm{A}$ & $\mathrm{H}$ & $\mathrm{C} 3^{9}$ & SED & GHlfp & 14,28 & 0,87 & NT & $\mathrm{X}$ & $\mathrm{X}$ & $\mathrm{X}$ & $\mathrm{X}$ & $\mathrm{X}$ & $\mathrm{X}$ \\
\hline $\begin{array}{l}\text { Leptochloa virgata (L.) P. } \\
\text { Beauv.* }\end{array}$ & A & $\mathrm{H}$ & $\mathrm{C} 4^{9}$ & DRE & - & 4,76 & 0,29 & NT & $\mathrm{X}$ & $\mathrm{X}$ & $\mathrm{X}$ & $\mathrm{X}$ & & $\mathrm{X}$ \\
\hline Lithachne horizontalis Chase & $\mathrm{F}$ & $\mathrm{U}$ & $\mathrm{C} 3^{12}$ & BDR & - & 4,76 & 0,29 & NT & & & & $\mathrm{X}$ & & \\
\hline $\begin{array}{l}\text { Luziola peruviana Juss. ex J.F. } \\
\text { Gmel. }\end{array}$ & $\mathrm{A}$ & $\mathrm{H}$ & $\mathrm{C} 3^{9}$ & $\begin{array}{l}\text { SAT, } \\
\text { SED }\end{array}$ & GHlfp & 4,76 & 0,29 & NT & & $\mathrm{X}$ & $\mathrm{X}$ & $\mathrm{X}$ & $\mathrm{X}$ & \\
\hline $\begin{array}{l}\text { Luziola spruceana Benth. ex } \\
\text { Döll }\end{array}$ & A & $\mathrm{H}$ & $\mathrm{C} 4^{6}$ & $\begin{array}{l}\text { SAT, } \\
\text { SED }\end{array}$ & - & 4,76 & 0,29 & NT & $\mathrm{X}$ & & & & & $\mathrm{X}$ \\
\hline $\begin{array}{l}\text { Megathyrsus maximus (Jacq.) } \\
\text { B.K. Simon \& S.W.L. Jacobs }\end{array}$ & A & $\mathrm{H}$ & $\mathrm{C}^{9}{ }^{\mathrm{T}}$ & $\begin{array}{l}\text { BDR, } \\
\text { DRE }\end{array}$ & SHufp & 14,28 & 0,87 & $\mathrm{NZ}$ & $\mathrm{X}$ & $\mathrm{X}$ & $\mathrm{X}$ & $\mathrm{X}$ & & $\mathrm{X}$ \\
\hline Melinis minutiflora P. Beauv. & $\mathrm{A}$ & $\mathrm{H}$ & $\mathrm{C} 4^{9}$ & BDR & - & 4,76 & 0,29 & NZ & $X$ & $\mathrm{X}$ & $\mathrm{X}$ & $\mathrm{X}$ & & \\
\hline Melinis repens (Willd.) Zizka & A & $\mathrm{H}$ & $\mathrm{C} 4^{9}$ & $\begin{array}{l}\text { BDR, } \\
\text { DRE }\end{array}$ & SHufp & 9,52 & 0,58 & $\mathrm{NZ}$ & & $\mathrm{X}$ & $X$ & $\mathrm{X}$ & & \\
\hline $\begin{array}{l}\text { Ocellochloa stolonifera (Poir.) } \\
\text { Zuloaga \& Morrone }\end{array}$ & $\mathrm{F}$ & $\mathrm{U}$ & $\mathrm{C} 3^{8}$ & DRE & - & 47,62 & 2,89 & NT & $\mathrm{X}$ & & & $\mathrm{X}$ & & $\mathrm{X}$ \\
\hline Olyra ciliatifolia Raddi* & $\mathrm{F}$ & $\mathrm{U}$ & $\mathrm{C} 3^{12}$ & $\begin{array}{l}\text { BDR, } \\
\text { DRE }\end{array}$ & Anl & 19 & 1,15 & NT & $\mathrm{X}$ & $\mathrm{X}$ & $\mathrm{X}$ & $\mathrm{X}$ & & $\mathrm{X}$ \\
\hline $\begin{array}{l}\text { Oplismenus hirtellus (L.) P. } \\
\text { Beauv. }\end{array}$ & $\mathrm{F}$ & $\mathrm{U}$ & $\mathrm{C} 3^{12}$ & DRE & - & 19 & 1,15 & NT & $\mathrm{X}$ & $\mathrm{X}$ & $\mathrm{X}$ & $X$ & & $\mathrm{X}$ \\
\hline Oryza latifolia Desv. & $\mathrm{A}$ & $\mathrm{H}$ & $\mathrm{C} 3^{12}$ & SED & GHlfp & 4,76 & 0,29 & NT & $\mathrm{X}$ & & $\mathrm{X}$ & $\mathrm{X}$ & & $\mathrm{X}$ \\
\hline
\end{tabular}




\begin{tabular}{|c|c|c|c|c|c|c|c|c|c|c|c|c|c|c|}
\hline \multirow{2}{*}{ Espécie } & \multirow{2}{*}{ FF } & \multirow{2}{*}{ EAL } & \multirow{2}{*}{$\mathbf{V F}$} & \multirow{2}{*}{ CHS } & \multirow{2}{*}{ UMV } & \multirow{2}{*}{$\begin{array}{c}\text { FA } \\
(\%)\end{array}$} & \multirow{2}{*}{$\begin{array}{l}\text { FR } \\
(\%)\end{array}$} & \multirow{2}{*}{ Status } & \multicolumn{6}{|c|}{ Domínio Fitogeográfico } \\
\hline & & & & & & & & & $\mathbf{A M}$ & CA & $\mathbf{C E}$ & MA & PP & PT \\
\hline $\begin{array}{l}\text { Otachyrium versicolor (Döll) } \\
\text { Henrard* }\end{array}$ & A & $\mathrm{H}$ & $\mathrm{C} 3^{12}$ & SAT & - & 9,52 & 0,58 & NT & $\mathrm{X}$ & & $\mathrm{X}$ & $\mathrm{X}$ & & \\
\hline Panicum condensatum Bertol. & A & E & $\begin{array}{l}\mathrm{C} 3 / \\
\mathrm{C} 4{ }^{11}\end{array}$ & SAT & - & 4,76 & 0,29 & NT & $\mathrm{X}$ & & $\mathrm{X}$ & $\mathrm{X}$ & & \\
\hline $\begin{array}{l}\text { Panicum dichotomiflorum } \\
\text { Michx.* }\end{array}$ & A & $\mathrm{H}$ & $\mathrm{C} 4^{12}$ & $\begin{array}{l}\text { SAT, } \\
\text { SED }\end{array}$ & GHlfp & 28,57 & 1,73 & NT & $\mathrm{X}$ & $\mathrm{X}$ & $\mathrm{X}$ & $\mathrm{X}$ & $\mathrm{X}$ & $\mathrm{X}$ \\
\hline Panicum millegrana Poir. & A & E & $\mathrm{C} 3^{9}$ & $\begin{array}{l}\text { DRE, } \\
\text { SED }\end{array}$ & GHlfp & 9,52 & 0,58 & NT & $\mathrm{X}$ & $\mathrm{X}$ & $\mathrm{X}$ & $\mathrm{X}$ & $\mathrm{X}$ & $\mathrm{X}$ \\
\hline Panicum repens $\mathrm{L} . *$ & A & $\mathrm{H}$ & $\mathrm{C} 4^{12}$ & SAT & GHlfp & 9,52 & 0,58 & $\mathrm{NZ}$ & $\mathrm{X}$ & $\mathrm{X}$ & $\mathrm{X}$ & $\mathrm{X}$ & & $\mathrm{X}$ \\
\hline Panicum trichanthum Nees* & A & $\mathrm{H}$ & $\mathrm{C} 3^{9}$ & $\begin{array}{l}\text { SAT, } \\
\text { SED }\end{array}$ & GHlfp & 9,52 & 0,58 & NT & $\mathrm{X}$ & $\mathrm{X}$ & $\mathrm{X}$ & $\mathrm{X}$ & $\mathrm{X}$ & $\mathrm{X}$ \\
\hline $\begin{array}{l}\text { Panicum tricholaenoides } \\
\text { Steud.* }\end{array}$ & A & $\mathrm{H}$ & $\mathrm{C} 4^{12}$ & $\begin{array}{l}\text { DRE, } \\
\text { SAT }\end{array}$ & - & 28,57 & 1,73 & NT & & & $\mathrm{X}$ & $\mathrm{X}$ & $\mathrm{X}$ & $\mathrm{X}$ \\
\hline $\begin{array}{l}\text { Parodiolyra micrantha } \\
\text { (Kunth) Davidse \& Zuloaga* }\end{array}$ & $\mathrm{F}$ & U & $\mathrm{C} 3^{12}$ & $\begin{array}{l}\text { BDR, } \\
\text { DRE }\end{array}$ & $A n l$ & 19 & 1,15 & NT & $\mathrm{X}$ & $\mathrm{X}$ & $\mathrm{X}$ & $\mathrm{X}$ & & \\
\hline Paspalum acuminatum Raddi & A & $\mathrm{H}$ & $\mathrm{C} 4^{12}$ & SAT & - & 28,57 & 1,73 & NT & & & $\mathrm{X}$ & $\mathrm{X}$ & $\mathrm{X}$ & $\mathrm{X}$ \\
\hline $\begin{array}{l}\text { Paspalum conjugatum P.J. } \\
\text { Bergius * }\end{array}$ & A & $\mathrm{H}$ & $\mathrm{C} 4^{9}$ & DRE & $A n l$ & 47,62 & 2,89 & NT & $\mathrm{X}$ & $\mathrm{X}$ & $\mathrm{X}$ & $\mathrm{X}$ & $\mathrm{X}$ & $\mathrm{X}$ \\
\hline $\begin{array}{l}\text { Paspalum conspersum } \\
\text { Schrad.* }\end{array}$ & A & $\mathrm{H}$ & $\mathrm{C} 4^{12}$ & DRE & SHmfp & 28,57 & 1,73 & NT & $\mathrm{X}$ & & $\mathrm{X}$ & $\mathrm{X}$ & & $\mathrm{X}$ \\
\hline $\begin{array}{l}\text { Paspalum falcatum Nees ex } \\
\text { Steud.* }\end{array}$ & A & $\mathrm{H}$ & $\mathrm{C} 4^{12}$ & DRE & - & 4,76 & 0,29 & NT & & & $\mathrm{X}$ & $\mathrm{X}$ & & \\
\hline $\begin{array}{l}\text { Paspalum fasciculatum Willd. } \\
\text { ex Flüggé }\end{array}$ & A & $\mathrm{H}$ & $\mathrm{C} 4^{9}$ & $\begin{array}{l}\text { DRE, } \\
\text { SAT, } \\
\text { SED }\end{array}$ & - & 9,52 & 0,58 & NT & $\mathrm{X}$ & & $\mathrm{X}$ & & & $\mathrm{X}$ \\
\hline Paspalum inaequivalve Raddi & A & $\mathrm{E}$ & $\mathrm{C} 4^{12}$ & $\mathrm{BDR}$ & - & 9,52 & 0,58 & NT & & & $\mathrm{X}$ & $\mathrm{X}$ & $\mathrm{X}$ & \\
\hline $\begin{array}{l}\text { Paspalum intermedium } \\
\text { Munro ex Morong \& Britton* }\end{array}$ & A & $\mathrm{H}$ & $\mathrm{C} 4^{12}$ & SAT & - & 38,09 & 2,31 & NT & & & $\mathrm{X}$ & $\mathrm{X}$ & $\mathrm{X}$ & $\mathrm{X}$ \\
\hline Paspalum notatum Flüggé* & A & $\mathrm{H}$ & $\mathrm{C} 4^{9}$ & BDR & - & 14,28 & 0,87 & NT & $\mathrm{X}$ & & $\mathrm{X}$ & $\mathrm{X}$ & $\mathrm{X}$ & \\
\hline Paspalum orbiculatum Poir.* & A & E & $\mathrm{C} 4^{9}$ & DRE & - & 4,76 & 0,29 & NT & $\mathrm{X}$ & $\mathrm{X}$ & $\mathrm{X}$ & $\mathrm{X}$ & $\mathrm{X}$ & \\
\hline $\begin{array}{l}\text { Paspalum repens P.J. } \\
\text { Bergius* }\end{array}$ & A & $\mathrm{H}$ & $\mathrm{C} 4^{9}$ & SED & GHlfp & 28,57 & 1,73 & NT & $\mathrm{X}$ & $\mathrm{X}$ & $\mathrm{X}$ & $\mathrm{X}$ & $\mathrm{X}$ & $\mathrm{X}$ \\
\hline Paspalum urvillei Steud. & A & $\mathrm{E}$ & $\mathrm{C} 4^{10}$ & SED & - & 9,52 & 0,58 & NT & & & $\mathrm{X}$ & $\mathrm{X}$ & $\mathrm{X}$ & \\
\hline $\begin{array}{l}\text { Phyllostachys aurea Carrière } \\
\text { ex Rivière \& C. Rivière }\end{array}$ & A & $\mathrm{H}$ & $\mathrm{C} 3^{12}$ & BDR & - & 4,76 & 0,29 & $\mathrm{NZ}$ & $\mathrm{X}$ & & $\mathrm{X}$ & $\mathrm{X}$ & & $\mathrm{X}$ \\
\hline Poа аппиа $\mathrm{L}$. & A & E & $\mathrm{C} 3^{9}$ & DRE & - & 4,76 & 0,29 & $\mathrm{NZ}$ & & & & $\mathrm{X}$ & $\mathrm{X}$ & \\
\hline $\begin{array}{l}\text { Reimarochloa acuta (Flüggé) } \\
\text { Hitchc. }\end{array}$ & A & $\mathrm{H}$ & $\mathrm{C} 4^{12}$ & SAT & - & 9,52 & 0,58 & NT & $\mathrm{X}$ & $\mathrm{X}$ & $\mathrm{X}$ & & & $\mathrm{X}$ \\
\hline
\end{tabular}




\begin{tabular}{|c|c|c|c|c|c|c|c|c|c|c|c|c|c|c|}
\hline \multirow{2}{*}{ Espécie } & \multirow{2}{*}{ FF } & \multirow{2}{*}{ EAL } & \multirow{2}{*}{ VF } & \multirow{2}{*}{ CHS } & \multirow{2}{*}{ UMV } & \multirow{2}{*}{$\begin{array}{c}\text { FA } \\
(\%)\end{array}$} & \multirow{2}{*}{$\begin{array}{l}\text { FR } \\
(\%)\end{array}$} & \multirow{2}{*}{ Status } & \multicolumn{6}{|c|}{ Domínio Fitogeográfico } \\
\hline & & & & & & & & & $\mathbf{A M}$ & CA & $\mathbf{C E}$ & MA & PP & PT \\
\hline $\begin{array}{l}\text { Rugoloa hylaeica Mez } \\
\text { (Zuloaga)* }\end{array}$ & $\mathrm{F}$ & $\mathrm{U}$ & $\mathrm{C} 4^{12}$ & SAT & - & 57,14 & 3,47 & NT & $\mathrm{X}$ & & $\mathrm{X}$ & $\mathrm{X}$ & & $\mathrm{X}$ \\
\hline $\begin{array}{l}\text { Rugoloa pilosa (Sw.) } \\
\text { Zuloaga* }\end{array}$ & $\mathrm{F}$ & $\mathrm{E}$ & $\mathrm{C} 3^{9}$ & SAT & - & 23,8 & 1,44 & NT & $\mathrm{X}$ & & & $\mathrm{X}$ & & \\
\hline $\begin{array}{l}\text { Rugoloa polygonata (Sw.) } \\
\text { Zuloaga* }\end{array}$ & $\mathrm{F}$ & $\mathrm{U}$ & $\mathrm{C} 3^{9}$ & $\begin{array}{l}\text { DRE, } \\
\text { SAT }\end{array}$ & - & 33,33 & 2,02 & NT & $\mathrm{X}$ & & $\mathrm{X}$ & $\mathrm{X}$ & & \\
\hline $\begin{array}{l}\text { Schizachyrium } \\
\text { microstachyum (Desv. ex } \\
\text { Ham.) Roseng. }\end{array}$ & A & $\mathrm{H}$ & $\mathrm{C} 4^{12}$ & DRE & $A n l$ & 4,76 & 0,29 & NT & $\mathrm{X}$ & & $\mathrm{X}$ & $\mathrm{X}$ & $\mathrm{X}$ & \\
\hline $\begin{array}{l}\text { Setaria parviflora (Poir.) } \\
\text { Kerguélen* }\end{array}$ & A & $\mathrm{H}$ & $\mathrm{C} 4^{9}$ & $\begin{array}{l}\text { BDR, } \\
\text { DRE }\end{array}$ & SHufp & 28,57 & 1,76 & NT & $\mathrm{X}$ & $\mathrm{X}$ & $\mathrm{X}$ & $\mathrm{X}$ & & $\mathrm{X}$ \\
\hline $\begin{array}{l}\text { Setaria vulpiseta (Lam.) } \\
\text { Roem. \& Schult.* }\end{array}$ & $\mathrm{F}$ & $\mathrm{E}$ & $\mathrm{C} 4^{9}$ & BDR & - & 23,8 & 1,44 & NT & $\mathrm{X}$ & $\mathrm{X}$ & $\mathrm{X}$ & $\mathrm{X}$ & & $\mathrm{X}$ \\
\hline $\begin{array}{l}\text { Sorghum halepense (L.) } \\
\text { Pers.* }\end{array}$ & A & $\mathrm{H}$ & $\mathrm{C} 4^{12}$ & $\mathrm{BDR}$ & - & 14,28 & 0,87 & $\mathrm{NZ}$ & $X$ & $\mathrm{X}$ & $\mathrm{X}$ & $X$ & & \\
\hline Sporobolus indicus (L.) R. Br. & A & $\mathrm{H}$ & $\mathrm{C} 4^{9}$ & $\begin{array}{l}\text { BDR, } \\
\text { DRE }\end{array}$ & $A n l$ & 23,8 & 1,44 & NT & $\mathrm{X}$ & $\mathrm{X}$ & $\mathrm{X}$ & $\mathrm{X}$ & $\mathrm{X}$ & $\mathrm{X}$ \\
\hline $\begin{array}{l}\text { Steinchisma laxum (Sw.) } \\
\text { Zuloaga* }\end{array}$ & A & $\mathrm{E}$ & $\begin{array}{l}\mathrm{C} 3 / \\
\mathrm{C} 4^{3}\end{array}$ & $\begin{array}{l}\text { DRE, } \\
\text { SAT }\end{array}$ & - & 57,14 & 3,47 & NT & $X$ & $X$ & $X$ & $X$ & & \\
\hline $\begin{array}{l}\text { Stephostachys mertensii } \\
\text { (Roth) Zuloaga \& Morrone* }\end{array}$ & A & E & $\begin{array}{l}\mathrm{C} 3 / \\
\mathrm{C} 4^{11}\end{array}$ & $\begin{array}{l}\text { DRE, } \\
\text { SAT }\end{array}$ & $\begin{array}{l}\text { Anl, } \\
\text { SHlfp }\end{array}$ & 38,09 & 2,31 & NT & $X$ & & $X$ & $X$ & & $X$ \\
\hline $\begin{array}{l}\text { Trichanthecium } \\
\text { schwackeanum (Mez) } \\
\text { Zuloaga \& Morrone* }\end{array}$ & $\mathrm{F}$ & $\mathrm{U}$ & $\mathrm{C} 3^{10}$ & $\begin{array}{l}\text { SAT, } \\
\text { SED }\end{array}$ & GHlfp & 14,28 & 0,87 & NT & X & $X$ & $X$ & $X$ & $X$ & $X$ \\
\hline $\begin{array}{l}\text { Urochloa arrecta (Hack. ex T. } \\
\text { Durand \& Schinz) Morrone } \\
\text { \& Zuloaga* }\end{array}$ & A & $\mathrm{H}$ & $\mathrm{C} 4^{3}$ & $\begin{array}{l}\text { SAT, } \\
\text { SED }\end{array}$ & GHlfp & 14,28 & 0,87 & $\mathrm{NZ}$ & $X$ & $\mathrm{X}$ & $X$ & $X$ & & \\
\hline $\begin{array}{l}\text { Urochloa brizantha*(A. } \\
\text { Rich.) R.D. Webster }\end{array}$ & A & $\mathrm{H}$ & $\mathrm{C} 4^{3}$ & $\begin{array}{l}\text { BDR, } \\
\text { DRE }\end{array}$ & - & 23,8 & 1,44 & $\mathrm{NZ}$ & $X$ & $X$ & $X$ & $X$ & $X$ & $X$ \\
\hline $\begin{array}{l}\text { Urochloa humidicola } \\
\text { (Rendle) Morrone \& } \\
\text { Zuloaga* }\end{array}$ & A & $\mathrm{H}$ & $\mathrm{C} 4^{3}$ & BDR & - & 14,28 & 0,87 & NZ & $X$ & X & $\mathrm{X}$ & $X$ & $X$ & \\
\hline $\begin{array}{l}\text { Urochloa mutica (Forssk.) } \\
\text { T.Q. Nguyen }\end{array}$ & A & $\mathrm{H}$ & $\mathrm{C} 4^{3}$ & $\begin{array}{l}\text { DRE, } \\
\text { SAT }\end{array}$ & - & 19 & 1,15 & NZ & X & $X$ & $X$ & $X$ & & \\
\hline $\begin{array}{l}\text { Urochloa plantaginea (Link) } \\
\text { R.D. Webster }\end{array}$ & A & $\mathrm{H}$ & $\mathrm{C} 4^{3}$ & $\begin{array}{l}\text { BDR, } \\
\text { DRE }\end{array}$ & - & 9,52 & 0,58 & NZ & $\mathrm{X}$ & $\mathrm{X}$ & $X$ & $X$ & $\mathrm{X}$ & \\
\hline
\end{tabular}

*PEVRI = Parque Estadual das Várzeas do Rio Ivinhema.

Referências bibliográficas: (1) Zuloaga et al. (1993); (2) Rossa et al. (1998); (3) Alliscioni (2000); (4) Graciano-Ribeiro (2002); (5) Alliscioni et al. (2003); (6) Camarão et al. (2003); (7) Silva \& Alquini (2003); (8) Sede et al. (2009); (9) Giraldo-Cañas (2010); (10) Zuloaga et al. (2011); (11) Morrone et al. (2012); (12) Filgueiras, dados não publicados. 
A vegetação com fisionomia florestal ocorre principalmente em diques marginais e nos paredões de arenito formação Caiuá, conforme estudos fitogeográficos realizados na área (Corradini et al. 2006; Stevaux et al. 2013). No dossel dos remanescentes florestais, foram registradas Bambusa tuldoides, Bambusa vulgaris, Dendrocalamus asper, Guadua chacoensis, Guadua paniculata e Guadua sp., das quais apenas as três espécies de Guadua são nativas, além de Gynerium sagittatum. No sub-bosque dos fragmentos florestais foram documentadas Acroceras zizanioides, Chusquea ramosissima, Dichanthelium surrectum, Hildaea pallens, Hymenachne amplexicaulis, Lasiacis ligulata, Lithachne horizontalis, Ocellochloa stolonifera, Olyra ciliatifolia, Oplismenus hirtellus, Parodiolyra micrantha, Rugoloa hylaeica, Rugoloa pilosa, Rugoloa polygonata e Setaria vulpiseta.

Quanto à exposição à luz, as espécies heliófilas foram as mais abundantes (65 spp. ou $69,15 \%$ ), seguidas pelas umbrófilas (16 spp. ou $17,02 \%$ ) e esciófilas (13 spp. ou 13,83\%). Além das áreas onde naturalmente ocorrem as espécies heliófilas, como as várzeas e o entorno dos corpos de água, o processo de ocupação desordenada da região no passado, com os intensos desmatamentos (Campos \& Souza 1997) e as cheias prolongadas, aumentaram as áreas de solo exposto (Hayakawa et al. 2010), o que provavelmente contribuiu para o elevado número de gramíneas heliófilas.

Quanto à via fotossintética, as gramíneas da PIAP estão assim distribuídas: C4 (58 spp. ou 61,7\%), C3 (30 spp. ou 31,91\%) e intermediárias C3/C4 (seis spp. ou 6,38\%). As espécies coletadas em ambientes campestres, naturais ou antropizados, são todas $\mathrm{C} 4$, já entre as coletadas nos ambientes florestais, 20 são $C 3$, nove são $C 4$ e três são $\mathrm{C} 3 / \mathrm{C} 4$. Entre as espécies encontradas ocupando estágios iniciais de sucessão em barras arenosas, lagoas temporárias e/ou locais alterados, 22 spp. $(23,4 \%)$ são $\mathrm{C} 4$, três spp. $(3,19 \%)$ são intermediárias $\mathrm{C} 3$ / C4 e duas spp. (2,13\%) são C3 (Tab. 1).

Estes dados estão de acordo com Osborne et al. (2014), segundo os quais a maioria das gramíneas tropicais apresenta via fotossintética C4. As plantas C3 ocorrem preferencialmente em ambientes úmidos ou sombreados (ACIESP 1997), em todas as latitudes, ao passo que as plantas C4 são comuns em ambientes semiáridos e quentes, preferencialmente nas áreas tropicais e subtropicais (Chapman \& Peat 1992). Essas diferenças fisiológicas estão, provavelmente, relacionadas à heterogeneidade dos ambientes da PIAP, promovendo condições ecológicas temporal e espacialmente distintas, que têm favorecido a maior abundância e prevalência de espécies $\mathrm{C} 4$ em relação às $\mathrm{C} 3$.

A maior parte das espécies foi coletada em substrato drenado (57 spp. ou $60,64 \%$ ), seguidas pelas espécies em substrato bem drenado (36 spp. ou $38,3 \%$ ), saturado ( 30 spp. ou $31,91 \%$ ) e sedimento (lama) (14 spp. ou 14,89\%). Considerando-se as espécies exclusivas de apenas um tipo de substrato, $22(23,4 \%)$ ocorrem em substrato drenado, 16 $(17,02 \%)$ ocorrem em substrato bem drenado, 11 $(11,7 \%)$ ocorrem em substrato saturado e quatro (4,25\%) ocorrem em sedimento (Tab. 1).

A Figura 1 apresenta um perfil esquemático com a distribuição de 14 espécies representativas dentro desses ambientes na PIAP. Os resultados indicam a predominância de espécies palustres (40 spp. ou 42,55\%), seguidas pelas terrestres (34 spp. ou 36,17\%) e aquáticas (20 spp. ou 21,28\%). Mais da metade das espécies estão relacionadas ao ambiente aquático e palustre (hidrófitas). Juntas estas classes perfazem $63,83 \%$ do total de espécies, principalmente, em sedimentos das margens das lagoas e outros corpos de água, substratos saturados e sujeitos à inundação. Esse fato sugere uma estreita relação de adaptação entre essas espécies e os regimes de inundações ocorrentes na PIAP. Segundo IBGE (2012), em terrenos aluviais a estrutura da comunidade vegetal reflete os efeitos das inundações sazonais.

Slusarski \& Souza (2012) realizaram uma avaliação temporal, com intervalo de aproximadamente doze anos, do sub-bosque de um remanescente florestal ripário da margem esquerda do Rio Paraná, município de Porto Rico, Paraná. Os autores observaram alterações estruturais e florísticas, com diminuição na densidade das espécies de áreas úmidas, dentre elas, as gramíneas Ocellochloa stolonifera (= Panicum stoloniferum) e Oplismenus hirtellus, dentre outras espécies fanerogâmicas, e aumento na densidade de espécies preferenciais de ambientes secos. Os autores sugerem que estes fatos constituam indícios de que as alterações provocadas pelo controle de nível da água do Rio Paraná, nesta planície, estejam afetando a vegetação marginal do remanescente estudado.

Entre as espécies aquáticas mais comuns no sedimento do entorno de lagoas e ambientes brejosos da várzea, destacam-se Dichanthelium surrectum, Luziola peruviana, Luziola spruceana, 
Otachyrium versicolor, Oryza latifolia, Panicum dichotomiflorum, Panicum trichanthum, Paspalum fasciculatum, Paspalum repens, Paspalum urvillei, Trichanthecium schwackeanum e Urochloa arrecta. Nos ambientes de solo úmido, saturado pela proximidade com os corpos de água ou pelo nível do lençol freático, foram amostradas Hymenachne amplexicaulis e Urochloa mutica. Fixas nos sedimentos, nas margens de corpos de água como lagoas ou rios semi-lóticos, ou ainda em locais de pouca correnteza dos rios, foram amostradas Panicum dichotomiflorum e Paspalum repens.

Ao redor das áreas rebaixadas, baixios (pântanos), e no entorno de lagoas com substrato encharcado e composto por sedimento, ocorrem espécies aquáticas ou higrófitas, tais como Echinochloa crus-galli, Echinochloa cruspavonis, Hymenachne amplexicaulis, Leersia hexandra, Luziola peruviana, Oryza latifolia, Panicum dichotomiflorum, Panicum millegrana, Panicum repens, Paspalum acuminatum, Paspalum fasciculatum, Paspalum repens, Paspalum urvillei, Rugoloa hylaeica, Rugoloa pilosa, Rugoloa polygonata, Trichanthecium schwackeanum e Urochloa arrecta.
Nos solos drenados mas sujeitos às inundações sazonais, como nos diques marginais, ocorrem espécies palustres como Coleataenia prionitis, Stephostachys mertensii e Paspalum conspersum. Nas áreas bem drenadas ocorrem espécies terrestres tais como Andropogon hypogynus, Setaria parviflora, Sporobolus indicus, dentre outras.

Os intervalos de Frequência Absoluta (FA) obtidos foram: 70,21\% (66 spp.) entre 0-20\%; $23,4 \%$ (22 spp.) entre $21-40 \% ; 5,32 \%$ (5 spp.) entre $41-60 \% ; 1,06 \%$ (1 sp.) entre 61-80\%. Hymenachne pernambucensis destacou-se como a espécie nativa mais frequente $(61,9 \%)$ e dominante nas lagoas da região (Souza et al. 2004b), ocorrendo em baixios alagados e no entorno das lagoas de várzea, tendo sido registrada em 13 das 21 localidades amostradas. Após esta espécie, aparecem Rugoloa hylaeica e Steinchisma laxum, ambas com 57,14\%, amostradas em 12 diferentes localidades (Tab. 1).

São comuns na PIAP: Guadua paniculata, Steinchisma laxum e Rugoloa hylaeica, todas de distribuição ampla nas áreas que compreendem as ilhas, a margem direita do Rio Paraná e os seus tributários, principalmente nos diques marginais inundáveis. Guadua paniculata, uma taboca nativa,

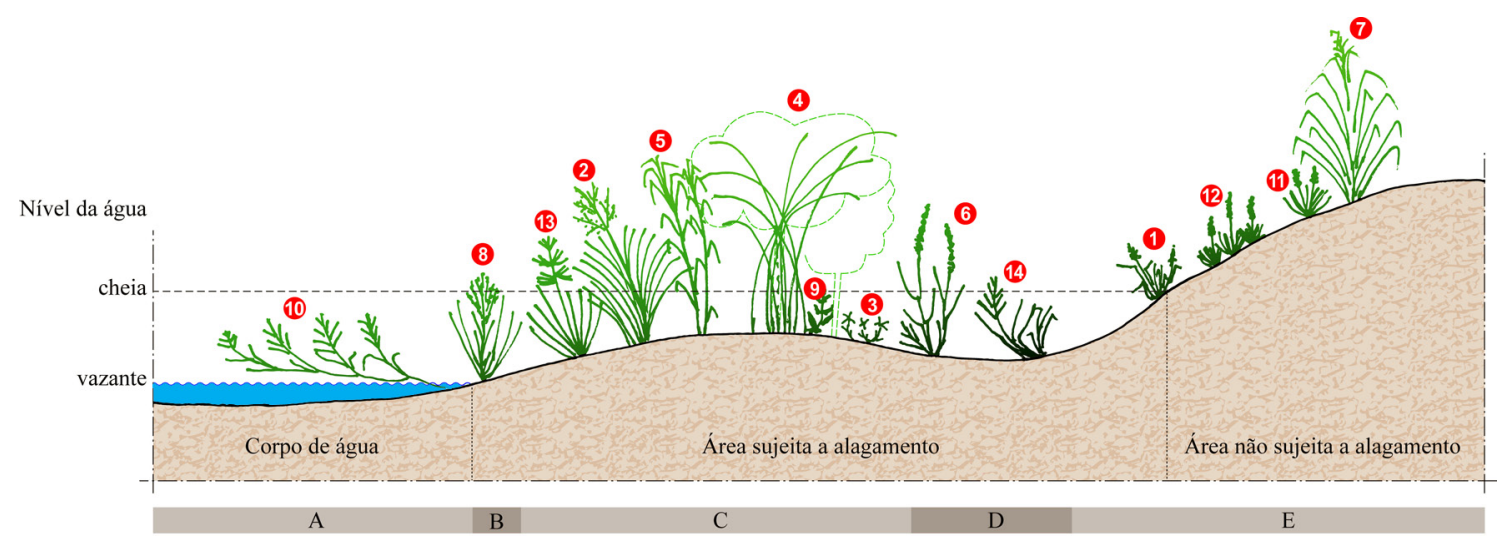

Figura 1 - Perfil esquemático da distribuição espacial de 14 espécies de Poaceae representativas da Planície de Inundação do Alto Rio Paraná e seu entorno, Mato Grosso do Sul e Paraná, Brasil, conforme o gradiente de condição topográfica e de inundação. Espécies: 1. Cenchrus echinatus; 2. Coleataenia prionitis; 3. Cynodon dactylon; 4. Guadua paniculata; 5. Gynerium sagittatum; 6. Hymenachne amplexicaulis; 7. Megathyrsus maximus; 8. Panicum dichotomiflorum; 9. Ocellochloa stolonifera; 10. Paspalum repens; 11. Setaria parviflora; 12. Sporobolus indicus; 13. Stephostachys mertensii; 14. Urochloa mutica ( $\mathrm{A}=$ sedimento; $\mathrm{B}=$ solo saturado pela proximidade com corpos de água superficiais; $\mathrm{C}=$ solo drenado; $\mathrm{D}=$ solo saturado pelo nível do lençol freático; $\mathrm{E}=$ solo bem drenado). Arte final: Jaime Luis Lopes/Nupélia/UEM (2014).

Figure 1 - Schematic profile of the spatial distribution of 14 representative species of Poaceae from the Upper Paraná River Floodplain and its environs, Mato Grosso do Sul and Paraná, Brazil, according to conditions of topographic and flooding gradients. Species: 1. Cenchrus echinatus; 2. Coleataenia prionitis; 3. Cynodon dactylon; 4. Guadua paniculata; 5. Gynerium sagittatum; 6. Hymenachne amplexicaulis; 7. Megathyrsus maximus; 8. Panicum dichotomiflorum; 9. Ocellochloa stolonifera; 10. Paspalum repens; 11. Setaria parviflora; 12. Sporobolus indicus; 13. Stephostachys mertensii; 14. Urochloa mutica. (A = sediment; B = soil saturated by proximity of superficial water bodies; C = drained soil; D $=$ soil saturated by groundwater level; $\mathrm{E}=$ soil well drainded). Final art: Jaime Luis Lopes/Nupélia/UEM (2014). 
é também amplamente distribuída em todo o Brasil (Londoño 2001).

Entre as espécies nativas coletadas em apenas uma localidade estão: Panicum condensatum, Leptochloa virgata, Lithachne horizontalis, Oryza latifolia, Paspalum acuminatum e Paspalum orbiculatum, das quais apenas Lithachne horizontalis não foi reencontrada (Fig. 2). Uma única coleta dessa espécie foi realizada há 25 anos em remanescente florestal do entorno da lagoa Finado Raimundo, na margem direita do Rio Ivinhema. Trabalhos de campo futuros serão necessários para tentar reencontrar esta espécie no PEVRI e na APA-IVRP.

Lithachne horizontalis encontra-se em perigo de extinção (Filgueiras et al. 2013) e Paspalum falcatum, ameaçada de extinção (Mamede et al. 2007; Filgueiras et al. 2013), portanto, estas espécies e suas áreas de ocorrência são prioritárias para conservação.

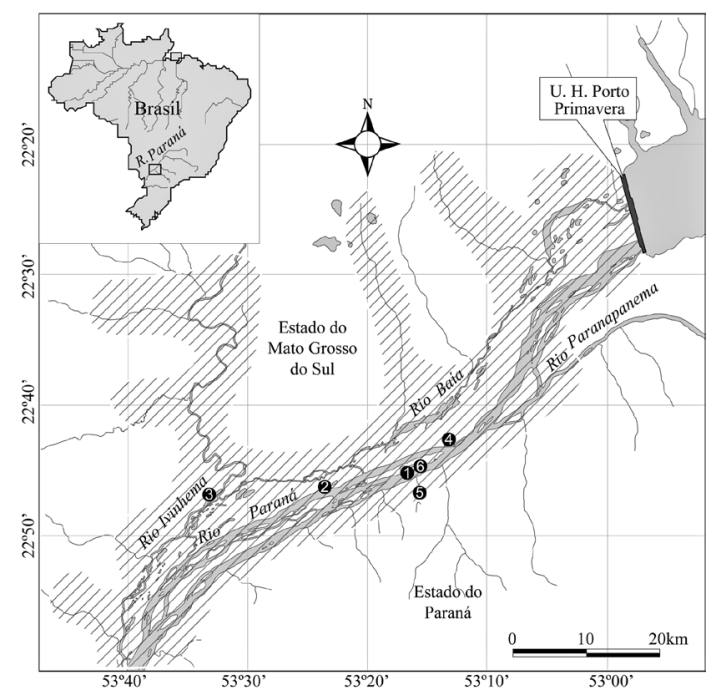

Figura 2 - Localização da área estudada. Locais de coleta de espécies de Poaceae nativas, localmente raras e coletadas em apenas uma localidade: 1. Panicum condensatum; 2. Leptochloa virgata; 3. Lithachne horizontalis; 4. Oryza latifolia; 5. Paspalum acuminatum; 6. Paspalum orbiculatum (áreas prioritárias para conservação). Planície de inundação do Alto Rio Paraná e entorno, Mato Grosso do Sul e Paraná, Brasil.

Figure 2 - Study site. Collection sites of native species of Poaceae, rare at the studied site and sampled just in one locality: 1. Panicum condensatum; 2. Leptochloa virgata; 3. Lithachne horizontalis; 4. Oryza latifolia; 5. Paspalum acuminatum; 6. Paspalum orbiculatum (priority areas for conservation). Upper Paraná River Floodplain and its surroundings, Mato Grosso do Sul and Paraná, Brazil.
Paspalum acuminatum, espécie nativa e localmente rara, foi coletada no leito assoreado do córrego Caracu, próximo a um bebedouro de bovinos, pastagem e cultura agrícola. Sua ocorrência em local altamente vulnerável indica que a espécie corre perigo de extinção local (Kawakita et al. 2016).

Paspalum falcatum, de ocorrência rara no alto Rio Paraná, com apenas duas coletas, é também citada como rara e exclusiva dos campos gerais do sul do Brasil (Smith et al. 1981). Segundo estes autores, Paspalum acuminatum é também considerada rara no sul do Brasil.

Em número de espécies destacaram-se as ilhas do Rio Paraná (47 spp. ou 50\% do total de espécies), o Rio Baía 41 spp. (43,62\% do total), o Rio Paraná em sua margem esquerda 40 spp. (42,55\% do total), o Rio Ivinhema $37 \mathrm{spp}$. (39,36\% do total) e o córrego Caracu $30 \mathrm{spp}$. $(31,91 \%$ do total). As ilhas que se destacaram em número de espécies, abrangem, como na várzea, diversos ambientes, tais como diques marginais, lagoas e ambientes brejosos.

Stevaux et al. (2013) classificaram a flora da PIAP em unidades de morfovegetação. A partir da compilação de dados dos rótulos das exsicatas e observações a campo, 33 espécies foram enquadradas nessa classificação (Tab. 1). Na Unidade de Morfo-Vegetação Anl [dique marginal] observou-se 16 espécies; em SHufp [Planície alta], sete espécies; em SHmfp [Planície Média], duas espécies; em GHlfp [Planície Baixa], 15 espécies.

A categoria Status, se nativa ou naturalizada, foi utilizada para avaliação do estado de conservação da área. A maior parte das espécies (64 ou 68,08\%) é nativa, o que indica a importância da área, apesar dos processos de antropização causado pela ocupação humana (Vazzoler et al. 1997; <http://www.peld.uem.br/>).

A Figura 3 apresenta o perfil graminosofitotopográfico esquemático transversal do Rio Paraná e imediações, com padrão de distribuição de sete espécies fitofisionomicamente representativas. Considerando-se os subsistemas da PIAP e seu entorno, foram encontradas 74 espécies para o SRPMD (canal Corutuba, canal Ipoitã, canal do Rio Baía, ilhota entre o canal Corutuba e o canal do Rio Baía, Rio Baía, Rio Curupaí, Rio Guiraí, Rio Ivinhema, Rio Paraná margem direita, Rio Samambaia e Rio Vitória), 41 para o SRPCI (Rio Paraná - barras arenosas e Rio Paraná - ilhas) e 59 para o SRPME (canal 
Cortado, córrego do Angico, córrego Caracu, córrego Nancy, riacho Piracema, ribeirão São Pedro, Rio Areia e Rio Paraná margem esquerda).

Nas barrancas de arenito da formação Caiuá e terraços baixos (Fig. 3), em áreas secas nunca inundadas ou naquelas inundadas em períodos recorrentes a cada cinco a sete anos com cheias extraordinárias (Souza-Filho \& Stevaux 2004), foram encontradas espécies terrestres nativas, como Setaria parviflora e Parodiolyra micrantha, além de outras introduzidas para pastagem, como Megathyrsus maximus, Urochloa spp., entre outras (Kawakita et al. 2016). Nas barras arenosas sujeitas a inundação sazonal, destacamse espécies terrestres: Axonopus leptostachyus, Cenchrus echinatus, Coleataenia prionitis, Digitaria violascens, Eragrostis articulata, Eragrostis bahiensis, Eragrostis ciliaris, Melinis repens e Urochloa mutica. Períodos mais longos de vazante ou de estiagem podem ainda favorecer o desenvolvimento de Axonopus suffultus e Urochloa brizantha, além de espécies pioneiras de outras famílias botânicas ou de estágios sucessionais posteriores. Entretanto, cheias intensas e de grande amplitude podem arrancar e transportar indivíduos, reiniciando o ciclo sucessional alhures. A distribuição diferenciada das espécies é um indicativo da relação da vegetação com os aspectos topográficos, fluviométricos, antropização na área, dentre outros.

Além das espécies terrestres, nas depressões alagadas ocorrem algumas higrófilas: Eriochloa punctata, Hymenachne amplexicaulis, Panicum dichotomiflorum, Paspalum conspersum, Rugoloa hylaeica e Steinchisma laxum. Nas "enseadas" a jusante das barras arenosas, o acúmulo de água forma ambientes que favorecem espécies palustres e hidrófitas, como Axonopus leptostachyus, Coleataenia prionitis, Eriochloa punctata, Hemarthria altissima, Hymenachne pernambucensis, Panicum dichotomiflorum, Paspalum conspersum, Rugoloa hylaeica e Urochloa mutica.

Das 94 espécies da PIAP, 63 (ou 67,02\%) também foram registradas para o Pantanal (Pott et al. 1986; Allem \& Valls 1987; Prado et al. 1994; Pott \& Pott 1997, 2000; Santos 2007; Pott et al. 2011).

Esta considerável porcentagem de espécies em comum deve-se, provavelmente, ao fato de que essas duas planícies de inundação, apesar das diferenças de dimensões de área e outros aspectos físicos e climáticos, fazem parte da rede hidrográfica da Bacia do Rio La Plata (Kawakita et al. 2016).

Digitaria violascens, Echinochloa crus-galli, Hymenachne amplexicaulis, Leersia hexandra, Megathyrsus maximus, Panicum condensatum, Panicum dichotomiflorum, Panicum trichanthym, Setaria parviflora e Steichisma laxum são espécies de ampla distribuição em áreas úmidas do Brasil (Junk 1970; Silva et al. 1983; Irgang et al 1984; Junk 1986; Pott et al. 1986; Allem \& Valls 1987; Oliveira et al. 1988; Pedralli et al. 1993a; Pedralli et al. 1993b; Prado et al. 1994; Irgang \& Gastal Júnior 1996; Gastal Júnior \& Irgang 1997; Pott \& Pott 1997; Rosa \& Irgang 1998; Pott \& Pott 2000; Bove et al. 2003; Rodrigues da Silva \& Filgueiras 2003; Santos 2007; Terneus 2007; Amaral et al. 2008; D'Angelo 2009; Rocha \& Lins 2009; Araújo et al. 2010; Alves et al. 2011; Pott et al. 2011; Medeiros et al. 2012; Ferreira et al. 2013; Moura Júnior et al. 2013; Oliveira et al. 2013; Rosseto \& Vieira 2013; Shirasuna et al. 2013; Bovini et al. 2014; Rodrigues \& Filgueiras 2014), na América do Sul (León \& Young 1996; Arbo \& Tressens 2005) ou ambientes associados ao Rio Paraná (Neiff 1986; Lewis et al. 1987; Stutz de Ortega 1987), tendo sido amostradas entre sete a nove regiões de estudo. Destas dez espécies, somente Steinchisma laxum também é comum na planície de inundação do alto Rio Paraná.

$\mathrm{Na}$ área do PEVRI foram encontradas 44 espécies, representando quase a metade do total de espécies da PIAP, apesar de a área do PEVRI ser de 73.315,15 ha. e o trecho superior da APAIVRP ser de 526.752 ha. Esse fato ressalta a importância dessa unidade de conservação, que apresenta grande diversidade de ambientes, boa parte deles bem preservados, tendo em vista a distância maior em relação aos núcleos urbanos e a restrição às atividades antrópicas.

A lagoa Figueira, um paleocanal localizado na ilha Porto Rico, pode secar totalmente nos ciclos plurianuais hipohídricos (Kita \& Souza 2003). Em pelo menos duas ocasiões, essa lagoa secou totalmente (1997 e 2001), formando gretas de contração no sedimento. Nessas gretas foi observada a presença maciça de Eragrostis hypnoides cobrindo parte do leito seco da lagoa. Em outro ponto da mesma ilha, Luziola spruceana, uma macrófita aquática, foi coletada durante os meses mais frios de 1998. Na lagoa Figueira, esta espécie foi novamente amostrada em 2001, também durante períodos de baixas temperaturas. 
O uso do solo na PIAP, no passado, para fins de pastagem bovina, favoreceu a introdução e disseminação de espécies exóticas e invasoras ( $c f$. Tab. 1). Nas porções mais elevadas das margens do Rio Paraná e nas ilhas, ocorria naturalmente a vegetação arbórea, que foi intensamente antropizada (Campos \& Souza 1997). Atualmente, estas porções são ocupadas por pastagens abandonadas, espécies exóticas e vegetação nativa em diferentes estágios de sucessão (Souza et al. 2009). Dentre as espécies exóticas destacam-se as africanas Megathyrsus maximus, Urochloa arrecta, $U$. brizantha, $U$. humidicola, U. mutica e U. plantaginea, que foram introduzidas no passado para fins de pastoreio bovino.

Foram observadas manchas (sensu Metzger 2001) de espécies exóticas, tais como Megathyrsus maximus, Urochloa brizantha, Urochloa humidicola, Urochloa mutica e Urochloa plantaginea, especialmente na margem esquerda do Rio Paraná e do Rio Ivinhema, e na margem direita do Rio Baía, em áreas antropizadas e locais de topografia mais elevada. Arundo donax, Cenchrus americanus, Cymbopogon nardus,
Cynodon nlemfuensis e Eragrostis tenella, embora introduzidas, foram observadas em apenas uma localidade cada, sem a formação de manchas. Melinis minutiflora é ainda pouco representativa na área, entretanto apresenta alto potencial invasor, principalmente em ambientes campestres (Kawakita et al. 2016). Urochloa arrecta foi registrada em lagoas da PIAP e em outros ambientes ripários da bacia do Rio Paraná (Mormul et al. 2010, Carniatto et al. 2013, Michelan et al. 2013). Diante disso, ressalta-se a necessidade da continuidade do monitoramento e manejo das populações de Urochloa arrecta, tendo em vista o seu potencial invasor em ambientes lênticos, principalmente em lagoas.

Com relação às espécies nativas, Hymenachne pernambucensis e Panicum tricholaenoides foram observadas formando manchas ao longo das várzeas do alto Rio Paraná, em áreas alagadas e sujeitas a inundação periódica e no entorno de lagoas do canal do Rio Baía. Hymenachne amplexicaulis forma manchas no entorno de lagoas rasas e pequenos corpos de água da várzea. Paspalum repens forma manchas nas margens dos canais secundários, nas

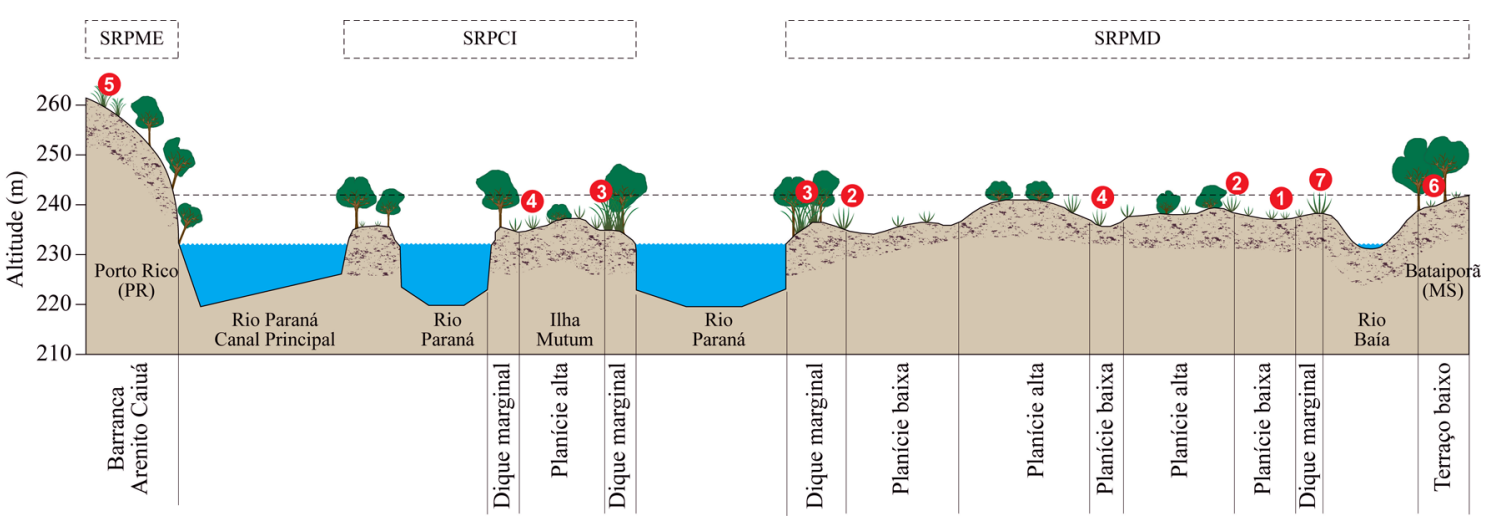

Figura 3 - Perfil graminoso-fitotopográfico transversal esquemático do Rio Paraná, ilhas e planície, demonstrando a distribuição de sete espécies fitofisionomicamente representativas na PIAP. Abreviações: SRPME = Subsistema Rio Paraná Margem Esquerda (terreno elevado, área não sujeita a alagamento); SRPCI = Subsistema Rio Paraná Central Ilhas; SRPMD = Subsistema Rio Paraná Margem Direita (planície de inundação, área sujeita a alagamento); linha tracejada = nível fluviométrico no período de cheia. Espécies encontradas nas Barrancas de Arenito Caiuá, no dique marginal, na planície (várzea) e no terraço baixo (vide Tab. 1): 1. Axonopus fissifolius;2. Coleataenia prionitis; 3. Guadua paniculata; 4. Hymenachne amplexicaulis; 5. Megathyrsus maximus; 6. Olyra ciliatifolia; 7. Panicum tricholaenoides. Fonte: modificado de Fachini (2001) e Stevaux et al. (2013). Arte final: Jaime Luiz Lopes/Nupélia/UEM (2014).

Figure 3 - Schematic cross-phytotopographic grassy profile of the Paraná River, islands and plain, depicting the distribution pattern of seven phytophysiognomically representative species of Poaceae in the PIAP. Acronyms: SRPME = Subsystem Rio Paraná Left Shore (high ground, not subject to flooding); SRPCI = Subsystem Rio Paraná Central Islands; SRPMD = Subsystem Rio Paraná Right Shore (floodplain area, subject to flooding); dash line = fluvial level in full period. Species found in the Barranca de Arenito Caiuá formations, levee, in plain (lowland), and in the lower terrace (see Tab. 1): 1. Axonopus fissifolius; 2. Coleataenia prionitis; 3. Guadua paniculata; 4. Hymenachne amplexicaulis; 5. Megathyrsus maximus; 6. Olyra ciliatifolia; 7. Panicum tricholaenoides. Source: modified from Fachini (2001) and Stevaux et al. (2013). Final art: Jaime Luiz Lopes/Nupélia/UEM (2014). 
lagoas, nas partes dos rios com corrente mais suave ou nos períodos de longa estiagem.

Guadua paniculata cresce ao longo dos diques marginais do canal Corutuba, do Rio Baía, ilhas e margens do Rio Paraná. Guadua chacoensis foi encontrada nas ilhas e nas margens do Rio Paraná e Rio Ivinhema, em diques marginais alagáveis ou alagados, formando touceiras com até cinco metros de largura e poucos colmos com cerca de $20 \mathrm{~m}$ de comprimento. Duas manchas de Guadua sp. foram observadas no dique marginal do entorno e no canal da lagoa das Garças, ambas situadas na margem direita do Rio Paraná. Na margem esquerda, foram observadas touceiras desta espécie com cinco a seis colmos.

Considerando a heterogeneidade ambiental e espacial de Poaceae na PIAP, estudos complementares avaliando a diversidade beta (Magurran 1988), são necessários.

\section{Considerações finais}

Este trabalho expõe a primeira contribuição para o estudo da distribuição das gramíneas na planície de inundação do alto Rio Paraná (PIAP). Os dados aqui demonstrados somam-se àqueles apresentados por Kawakita et al. (2016), e representam um incremento no conhecimento da composição e estrutura da flora agrostológica da PIAP.

Os resultados sugerem que fatores antrópicos incidem e afetam substancialmente o padrão de distribuição, a densidade e a diversidade de espécies na PIAP. Alterações antropogênicas locais (e.g., represamentos e degradação da vegetação ripária) e globais (e.g., alterações climáticas, mudanças dos regimes sazonais de chuvas, etc.) interferem diretamente na dinâmica aluvial e, por consequência, na estrutura de toda a vegetação da PIAP. Embora as áreas próximas às zonas antropizadas e locais de topografia mais elevada sofram maior pressão (Kawakita et al. 2016), as áreas mais baixas tendem a sofrer mais com o carreamento de resíduos, degradação da vegetação ripária, erosão, assoreamento, além de flutuações acentuadas e recorrentes no nível e na qualidade da água do rio. De fato, parte dos resultados aqui apresentados são indicativos de que, embora protegidas, várias localidades da PIAP ainda permanecem vulneráveis à ação antrópica.

A introdução e a disseminação de espécies exóticas, especialmente da macrófita aquática Urochloa arrecta, deve ser também encarada como ponto chave para as ações de conservação e manejo, tanto na APA-IVRP quando no PEVRI e, dessa forma, evitar a perda da diversidade local.

Apesar dos fatores antrópicos do passado e atuais, a ocorrência de espécies nativas, raras ou em perigo de extinção (Kawakita et al. 2016) agrega ainda maior valor à PIAP, principalmente sob o ponto de vista da conservação. Neste contexto, estudos em outras localidades ainda escassamente amostradas da PIAP são necessários e podem oferecer novas informações acerca desta e de outras questões. Estas medidas contribuirão para a tomada de decisões para a conservação da biodiversidade desta APA, de acordo com os objetivos e metas da Estratégia Global de Conservação de Plantas no Brasil (Dias \& Hoft 2013).

O conhecimento da diversidade de plantas herbáceas (incluindo a diversidade genética) é de fundamental importância para o entendimento da diversidade ecológica desse ecossistema e para seu uso sustentável. Dessa forma, conhecer o padrão de distribuição, a diversidade, a abundância e a ecologia desses organismos permite acompanhar e inferir sobre as relações e as alterações naturais e/ou antrópicas, capazes de interferir em toda a cadeia ecológica presente na PIAP.

\section{Agradecimentos}

À Doutora Maria Conceição de Souza (PGB/ PEA/DBi/UEM), o apoio logístico e administrativo; aos Doutores Pedro Lage Viana, Mauro Parolin, Evanilde Benedito, Simone Slusarski, Erivelton Goulart, José Cândido Stevaux e Maria Margarida da Rocha Fiuza de Melo, as valiosas sugestões; à equipe do Laboratório de Vegetação Ripária do Nupélia (Mata Ciliar), em especial, ao Carlos Eduardo Bento Fernandes, a inestimável ajuda em campo e no laboratório; ao Nupélia/UEM, PEA/UEM e ao Núcleo de Pesquisa Curadoria do Herbário do Instituto de Botânica de São Paulo, o apoio logístico; ao PGB/UEM e ao CNPq/PELD, o auxílio financeiro parcial. RSR agradece ao CNPq e a CAPES, respectivamente, as bolsas de Mestrado e Doutorado concedidas.

\section{Referências}

ACIESP - Academia de Ciências do Estado de São Paulo (1997) Glossário de ecologia. 2a ed. Vol. 103. CNPq, FINEP, Academia de Ciências, São Paulo. 352p.

Agostinho AA, Rodrigues L, Gomes LC, Thomaz SM \& Miranda LE (2004) Structure and functioning of the Paraná river and its floodplain. LTER - site 6 (PELD Sítio 6). Editora da Universidade Estadual de Maringá, Maringá. 275p. 
Allem AC \& Valls JFM (1987) Recursos forrageiros nativos do pantanal mato-grossense. EMBRAPA, Brasília. 339p.

Alliscioni SS (2000) Anatomia ecológica de algumas espécies del género Paspalum (Poaceae, Panicoideae, Paniceae). Darwiniana 38: 187-207.

Alliscioni SS, Giussani LM, Zuloaga FO \& Kellog EA (2003) A molecular phylogeny of Panicum (Poaceae: Paniceae): tests of monophyly and phylogenetic placement within the Panicoideae. American Journal of Botany 90: 796-821.

Alves JAA, Tavares AS \& Trevisan R (2011) Composição e distribuição de macrófitas aquáticas na lagoa da restinga do Massiambu, área de proteção ambiental entorno costeiro, SC. Rodriguésia 62: 785-801.

Amaral DD, Prost MT, Bastos MNC, Costa Neto SV \& Santos JUM (2008) Restingas do litoral amazônico, estados do Pará e Amapá, Brasil. Boletim Museu Paraense Emílio Goeldi, Ciências Naturais 3: 35-67.

Araújo RB, Langeani F \& Ranga NT (2010) Vascular plants of oxbow lakes of Turvo River, Upper Paraná River basin, São Paulo State, Brazil. Check List 6: 58-61.

Arbo MM \& Tressens SG (eds.) (2005) Flora Del Iberá. Reimpresión 2005. Eudene, Corrientes. 613p. ISBN 950-656062-5.

BFG - The Brazil Flora Group (2015) Growing knowledge: an overview of seed plant diversity in Brazil. Rodriguésia 66: 1085-1113.

Boldrini II, Longhi-Wagner HM \& Boechat SC (2008) Morfologia e taxonomia de gramíneas Sul-RioGrandenses. Editora da Universidade Federal do Rio Grande do Sul, Porto Alegre. 87p.

Bove CP, Gil ASB, Moreira CB \& Anjos RFB (2003) Hidrófitas fanerogâmicas de ecossistemas aquáticos temporários da planície costeira do estado do Rio de Janeiro, Brasil. Acta Botanica Brasilica 17: 119-135.

Bovini MG, Faria M, Oliveira RR \& Kurtz BC (2014) Floristic diversity of the Cagarras Islands Natural Monument, Rio de Janeiro, Brazil. Check List 10: 366-373.

Braun-Blanquet J (1979) Fitosociologia: bases para el estúdio de las comunidades vegetales. Ed. H. Blume, Madri. 820p.

Camarão AP, Marques JRF, Martinez GB, Lopes CAC, Costa NA, Lourenço-Júnior JB, Carvalho NN, Pimentel ES \& Filho RN (2003) Recursos forrageiros na várzea. In: Marques JRF, Lopes CAC \& Martinez GB (eds.) Produção animal nas várzeas do Rio Amazonas. EMBRAPA Amazônia Oriental, Belém. Pp. 255-301.

Campos JB (2004) Spatial characterization of the vegetation. In: Thomaz SM, Agostinho AA \& Hahn NS (eds.) The upper Paraná river and its floodplain: physical aspects, ecology and conservation. Backhuys Publishers, Leiden. Pp. 369-380.
Campos JB \& Souza MC (1997) Vegetação. In: Vazzoler AEA, Agostinho AA \& Hahn NS (eds.) A planície de inundação do alto rio Paraná: aspectos físicos, biológicos e socioeconômicos. Editora da Universidade Estadual de Maringá, Maringá. Pp. 331-342.

Carniatto N, Thomaz SM, Cunha ER, Fugi R \& Ota RR (2013) Effects of an invasive alien Poaceae on aquatic macrophytes and fish communities in a Neotropical reservoir. Biotropica 45: 747-754.

Chapman GP \& Peat WE (1992) An introduction to the grasses: including bamboos and cereals. CAB International, Wallingford. 111p.

Corradini FA (2006) Processos de conectividade e a vegetação ripária do alto Rio Paraná - MS. Dissertação de Mestrado. Universidade Estadual de Maringá, Maringá. 80p.

Corradini FA, Fachini MP \& Stevaux JC (2006) Controle geomorfológico da distribuição da vegetação ripária do Rio Paraná: Parte I - unidades geomórficas da planície de inundação. Revista Universidade de Guarulhos, Geociências 5: 13-21.

Corradini FA, Stevaux JC \& Fachini MP (2008) Geomorfologia e distribuição da vegetação ripária na Ilha Mutum, Rio Paraná - PR/MS. Geociências 27: 345-354.

D’Angelo SA (2009) Colonização vegetal em áreas de sedimentação recente na várzea da Amazônia Central. Dissertação (Mestrado em Ciências Biológicas - Botânica), Instituto Nacional de Pesquisas da Amazônia, Manaus. 101p.

Dias BFS \& Hoft R (2013) Desafios para a implementação da estratégia global para a conservação de plantas no Brasil. In: Martinelli G, Moraes MA (orgs.) Livro Vermelho da Flora do Brasil. Andrea Jakobsson Estúdio: Instituto de Pesquisas Jardim Botânico do Rio de Janeiro, Rio de Janeiro. Pp. 26-39.

Fachini MP (2001) Fitofisionomia e levantamento florístico em transectos na planície de inundação do alto Rio Paraná (PR e MS). Dissertação de Mestrado. Universidade Estadual de Maringá, Maringá. 36p.

Ferreira EVR, Prata APN \& Mello AA (2013) Floristic list from a Caatinga Remnant in Poço Verde, Sergipe, Brazil. Check List 9: 1354-1360.

Filgueiras TS (1990) Gramíneas africanas: introdução no Brasil. Cadernos de Geociências 5: 57-63.

Filgueiras TS, Oliveira RP, Sfair JC, Monteiro NP \& Borges RAX (2013) Poaceae. In: Martinelli G \& Moraes MA (orgs.) Livro Vermelho da Flora do Brasil. Andrea Jakobsson Estúdio: Instituto de Pesquisas Jardim Botânico do Rio de Janeiro, Rio de Janeiro. Pp. 858-881.

Fragal EH, Alves FC \& Souza-Filho EE (2012) A influência da inundação na dinâmica da vegetação da planície fluvial do Alto Rio Paraná em 2007. Revista Brasileira de Geomorfologia 13: 299-310. 
Gastal Júnior CV.S \& Irgang BE (1997) Levantamento de macrófitas aquáticas do Vale do Rio Pardo, Rio Grande do Sul. Iheringia série Botânica 49: 1-88.

Giraldo-Cañas D (2010) Distribución e invasión de gramíneas C3 y C4 (Poaceae) en un gradiente altitudinal de los Andes de Colombia. Caldasia 32: 65-86.

Graciano-Ribeiro D (2002) Caracteres anatômicos aplicados à taxonomia e variações ambientais das espécies de Otachyrium (Poaceae: Panicoideae) da América do Sul. Tese de Doutorado. Universidade de São Paulo, São Paulo. 215p.

Hayakawa EH, Couto EV, Souza-Filho EE, Prado BR \& Paula PF (2010) Análise temporal da planície de inundação do Alto Rio Paraná (região de Porto Rico, PR) através de dados de sensoriamento remoto. Boletim de Geografia 28: 115-126.

IBGE - Instituto Brasileiro de Geografia e Estatística (2012) Manual técnico da vegetação brasileira. Vol. 1. $2^{\mathrm{a}}$ ed. Manuais técnicos em Geociências. Instituto Brasileiro de Geografia e Estatística, Rio de Janeiro. 271p.

Irgang BE \& Gastal Júnior CVS (1996) Macrófitas aquáticas da planície costeira do RS. Editora UFRGS, Porto Alegre. 290p.

Irgang BE, Pedralli G \& Waechter JL (1984) Macrófitos aquáticos da Estação Ecológica do Taim, Rio Grande do Sul, Brasil. Roessléria 6: 395-404.

Junk WJ (1970) Investigations on the ecology and production-biology of the "floating meadows" (Paspalo-Echinochloetum) on the Middle Amazon. I. The floating vegetation and its ecology. Amazoniana 2: 449-495.

Junk WJ (1986) Aquatic plants of the Amazon system. In: Davies BR \& Walker KF (eds.) The ecology of river systems. Dr. W. Junk Publishers, Dordrecht. Pp. 319-337.

Junk WJ, Bayle PB \& Sparks RE (1989) The flood pulse concept in riverfloodplain systems. In: Dodge DP. Proceedings of the International Large River Symposium (LARS). Department of Fisheries and Oceans, Ottawa. Vol. 106. Canadian Special Publication of Fisheries and Aquatic Science, Ottawa. Pp. 110-127.

Kawakita K, Rodrigues RS \& Filgueiras TS (2016) Poaceae em uma planície de inundação no Brasil: listagem florística e novas ocorrências. Hoehnea 43: 203-216.

Kita KK \& Souza MC (2003) Levantamento florístico e fitofisionomia da lagoa Figueira e seu entorno, planície alagável do alto Rio Paraná, Porto Rico, estado do Paraná, Brasil. Acta Scientiarum 25: 145-155.

León B \& Young KR (1996) Aquatic plants of Peru: diversity, distribution and conservation. Biodiversity and Conservation 5: 1169-1190.

Lewis JP, Francheschi EA \& Prado DE (1987) Effects of extraordinary floods on the dynamics of tall grasslands of the river Paraná valley. Phytocoenologia 15: 235-251.
Londoño X (2001) Guadua. In: Wanderley MGL, Shepherd GJ \& Giulietti AM (coords.) Flora fanerogâmica do estado de São Paulo. Instituto de Botânica, São Paulo. Vol. 1, pp. 38.

Magurran AE (1988) Ecological diversity and its measurement. Chapman and Hall, London. 179p.

Mamede MCH, Souza VC, Prado J, Barros F, Wanderley MGL \& Rando JG (org.) (2007) Livro vermelho das espécies vegetais ameaçadas do estado de São Paulo. Vol. 1. Instituto de Botânica, Imprensa Oficial, São Paulo, 165p.

Medeiros MB, Walter BMT, Silva GP, Gomes BM, Lima ILP, Moser P, Oliveira WL \& Cavalcanti TB (2012) Vascular flora of the Tocantins River Middle Basin, Brazil. Check List 8: 852-885.

Metzger JP (2001) O que é ecologia de paisagens? Biota Neotropica 1: 1-9.

Michelan TS, Thomaz SM \& Bini LM (2013) Native macrophyte density and richness affect the invasiveness of a tropical Poaceae species. Plos One 8: e60004.

Mormul RP, Ferreira FA, Michelan TS, Carvalho P, Silveira MJ \& Thomaz SM (2010) Aquatic macrophytes in the large, sub-tropical Itaipu Reservoir, Brazil. Revista de Biologia Tropical 58: 1437-1452.

Morrone O, Aagesen L, Scataglini MA, Salariato DL, Denham SS, Chemisquy MA, Sede SM, Giussani LM, Kellogg E \& Zuloaga FO (2012) Phylogeny of the Paniceae (Poaceae: Panicoideae): integrating plastid DNA sequences and morphology into a new classification. Cladistics 28: 333-356.

Moura-Júnior EG, Lima LF, Silva SSL, Paiva RMS, Ferreira FA, Zickel CS \& Pott A (2013) Aquatic macrophytes of northeastern Brazil: checklist, richness, distribution and life forms. Check List 9: 298-312.

Neiff JJ (1986) Aquatic plants of the Paraná system. In: Davies BR \& Walker KF (eds.) The ecology of river systems. Series Editor HJ Dumont, Junk, Dordrecht. Pp. 557-571.

Oliveira MLAA, Neves MTMB, Strehl T, Ramos RLD \& Bueno OL (1988) Vegetação de macrófitos aquáticos das nascentes do Rio Gravataí (Banhado Grande e Banhado Chico Lomã), Rio Grande do Sul, Brasil - levantamento preliminar. Iheringia, Série Botânica: 67-80.

Oliveira RC, Silva AS, Ribeiro ARO, Araújo JE, Oliveira OF \& Camacho RGV (2013) Angiosperm species of the riparian vegetation of the Apodi-Mossoró River, Rio Grande do Norte, Brazil. Check List 9: 740-751.

Oliveira RP \& Longhi-Wagner HM (2005) Olyra bahiensis (Poaceae - Olyreae): uma nova espécie para a mata atlântica do estado da Bahia, Brasil. Revista Brasileira de Botânica 28: 835-839.

Osborne CP, Anna Salomaa A, Kluyver TA, Visser V, Kellogg EA, Morrone O, Vorontsova MS, Clayton WD \& Simpson DA (2014) A global database of 
C4 photosynthesis in grasses. New Phytologist 204: 441-446.

Pedralli G, Meyer ST, Teixeira MC \& Stehmann JR (1993a) Levantamento dos macrófitos aquáticos e da mata ciliar do reservatório de Volta Grande, Minas Gerais, Brasil. Iheringia, Série Botânica: 29-40.

Pedralli G, Stehmann JR, Teixeira MC, Oliveira VL \& Meyer ST (1993b) Levantamento da vegetação aquática ("macrófitos") na área da EPDA-Peti, Santa Bárbara, MG. Iheringia, Série Botânica: 15-28.

Pott VJ \& Pott A (1997) Checklist das macrófitas aquáticas do pantanal, Brasil. Acta Botanica Brasilica 11: 215-227.

Pott VJ \& Pott A (2000) Plantas Aquáticas do Pantanal. Embrapa, comunicação para transferência de tecnologia, Brasília. 404p.

Pott VJ, Rego SCA \& Pott A (1986) Plantas uliginosas e aquáticas do Pantanal arenoso. Pesquisa em andamento. $N^{\circ}$ 6. Embrapa-CPAP, Corumbá. Pp. 1-12.

Pott VJ, Pott A, Lima LCP, Moreira SN \& Oliveira AKM (2011) Aquatic macrophyte diversity of the Pantanal wetland and upper basin. Brazilian Journal of Biology, 71 (1, suppl.): 255-263.

Prado AL, Heckman CW \& Martins FR (1994) The seasonal succession of biotic communities in wetlands of the tropical wet-and-dry climatic zone: II. The aquatic macrophyte vegetation in the Pantanal of Mato Grosso, Brazil. International Review of Hydrobiology 79: 569-589.

Rocha AES \& Lins ALFA (2009) Checklist das Poaceae de áreas inundáveis e inundadas do nordeste do estado do Pará. Acta Amazonica 39: 763-772.

Rocha PC \& Comunello E (2009) Geomorfologia e áreas inundáveis na planície fluvial do alto Rio Paraná. Anais do $13^{\circ}$ Simpósio Brasileiro de Geografia Física Aplicada, Viçosa. Pp. 60-75.

Rodrigues RS \& Filgueiras TS (2014) A tribo Paniceae s.l. (Poaceae: Panicoideae) na Reserva Biológica de Mogi-Guaçu, SP, Brasil. Hoehnea 41: 589-622.

Rodrigues-da-Silva R \& Filgueiras TS (2003) Gramíneas (Poaceae) da Área de Relevante Interesse Ecológico (ARIE) "Santuário De Vida Silvestre Do Riacho Fundo", Distrito Federal, Brasil. Acta Botanica Brasilica 17: 467-486.

Rosa FF \& Irgang BE (1998) Comunidades vegetais de um segmento da planície de inundação do Rio dos Sinos, Rio Grande do Sul, Brasil. Iheringia, Série Botânica 50: 75-87.

Rossa B, Tu Aers AV, Naidoo G \& Von Willert DJ (1998) Arundo donax L. (Poaceae): a C3 species with ununsually high photosynthetic capacity. Acta Botanica 111: 216-221.

Rosseto EFS \& Vieira AOS (2013) Vascular flora of the Mata dos Godoy State Park, Londrina, Paraná, Brazil. Check List 9: 1020-1034.

Santos CAG (2007) Revisão de Axonopus serie Suffulti G.A.Black (Poaceae: Paniceae) para o Brasil. Tese de
Doutorado em Ciências, área de Botânica. Instituto de Biociências, Universidade de São Paulo, São Paulo. 165p.

Schmidt R \& Longhi-Wagner HM (2009) A tribo Bambuseae (Poaceae, Bambusoideae) no Rio Grande do Sul, Brasil. Revista Brasileira de Biociências 7: 71-128.

Sede S, Zuloaga FO \& Morrone O (2009) Phylogenetics studies in the Paniceae (Poaceae - Panicoideae): Ocellochloa a new genus from the New World. Systematic Botany 34: 684-692.

Shirasuna RT, Filgueiras TS \& Barbosa LM (2013) Poaceae do Rodoanel Mario Covas, trecho sul, São Paulo, SP, Brasil: florística e potencial de uso na restauração de áreas degradadas. Hoehnea 40: 521-536.

Silva LM \& Alquini Y (2003) Anatomia comparativa de folhas e caules de Axonopus scoparius (Flügge) Kuhlm. e Axonopus fissifolius (Raddi) Kuhlm. (Poaceae). Revista Brasileira de Botânica 26: 185192.

Silva TS, Sendulsky T \& Burman AG (1983) Flora Fanerogâmica da Reserva do Parque Estadual das Fontes do Ipiranga, São Paulo, Brasil: 176 Gramineae (Poaceae). Hoehnea 10: 58-73.

Slusarski SR \& Souza MC (2012) Inventário florístico ampliado na mata do Araldo, planície de inundação do alto Rio Paraná, Brasil. Revista de Estudos Ambientais 14: 14-27.

Smith LB, Wasshausen DC \& Klein RM (1981) Gramíneas: 1. Bambusa até 44. Chloris. In: Reitz R (ed.) Flora Ilustrada Catarinense. Herbário Barbosa Rodrigues, Itajaí. $435 \mathrm{p}$.

Souza MC, Cislinski J \& Romagnolo MB (1997) Levantamento florístico. In: Vazzoler AEAM, Agostinho AA \& Hahn NS (eds.) A planície de inundação do alto Rio Paraná: aspectos físicos, biológicos e socioeconômicos. Editora da Universidade Estadual de Maringá, Maringá. Pp. 343-368.

Souza MC, Kawakita K, Slusarski SR \& Pereira GF (2009) Vascular flora of the Upper Paraná River floodplain. Brazilian Journal of Biology 69: 735-745.

Souza MC, Kita KK, Romagnolo MB, Tomazini V, Albuquerque EC, Secorun AC \& Miola DTB (2004a) Riparian vegetation of the upper Paraná river floodplain, Paraná and Mato Grosso do Sul States, Brazil. In: Agostinho AA, Rodrigues L, Gomes LC, Thomaz SM \& Miranda LE (eds.) Structure and functioning of the Paraná river and its floodplain: LTER - Site 6 - (PELD Sítio 6). Editora da Universidade Estadual de Maringá, Maringá. Pp. 233-238.

Souza MC, Romagnolo MB \& Kita KK (2004b) Riparian vegetation: ecotones and plant communities. In: Thomaz SM, Agostinho AA \& Hahn NS (eds.) The upper Paraná River and floodplain: physical aspects, ecology and 
conservation. Backhuys Publishers, Leiden. Pp. 353-367.

Souza-Filho EE (1994) Feições do sistema anastomosado pré-atual do Rio Paraná. In: Anais do $38^{\circ}$ Congresso Brasileiro de Geologia. SBG, Camboriú. Pp. $407-$ 409.

Souza-Filho EE \& Stevaux JC (2004) Geology and geomorphology of the Baía-Curutuba-Ivinhema River Complex. In: Thomaz SM, Agostinho AA \& Hahn NS (eds.) The upper Paraná river and its floodplain: physical aspects, ecology and conservation. Backhuys Publishers, Leiden. Pp $1-24$.

Stevaux JC \& Souza-Filho EE (1997) Geologia e geomorfologia do complexo Rio Baía, Curutuba, Ivinhema. In: Vazzoler AEAM, Agostinho AA \& Hahn NS (eds.) A planície de inundação do alto Rio Paraná: aspectos físicos, biológicos e socioeconômicos. Editora da Universidade Estadual de Maringá, Maringá. Pp. 3-46.

Stevaux JC, Corradini FA \& Aquino S (2013) Connectivity processes and riparian vegetation of the upper Paraná River, Brazil. Journal of South American Earth Sciences 46: 113-121.

Stutz de Ortega LC (1987) Etudes floristiques de divers stades secondaires des formations forestiès du Haut Parana (Paraguay Oriental). Structure, composition floristique et régénération naturalle: comparaison entre la florêt primaire et la forêt sélectivement exploitée. Candollea 42: 205-262.
Terneus E (2007) Las plantas acuáticas en el sistema lacustre-riberino Lagartococha, Reserva De Producción Faunística Cuyabeno, Ecuador. Actualidades Biológicas 29: 97-106.

UEM - Universidade Estadual de Maringá (2017) A planície alagável do Rio Paraná: estrutura e processo ambiental. Nupélia/PELD, Maringá. Disponível em $<$ http://www.peld.uem.br/>. Acesso em 17 maio 2017.

Vazzoler AEA, Agostinho AA \& Hahn NS (eds.) (1997) A planície de inundação do alto Rio Paraná: aspectos físicos, biológicos e socioeconômicos. Editora da Universidade Estadual de Maringá, Maringá. 460p.

Watson L \& Dallwitz MJ (1992) The grass genera of the world: descriptions, illustrations, identification, and information retrieval; including synonyms, morphology, anatomy, physiology, phytochemistry, cytology, classification, pathogens, world and local distribution, and references. Disponível em $<$ http:// delta-intkey.com/angio/www/graminea.htm $>$. Acesso em 20 dezembro 2012.

Zuloaga FO, Ellis RP \& Morrone O (1993) A revision of Panicum subg. Dichanthelium (Poaceae: Panicoideae: Paniceae) in Mesoamerica, the West Indies and South Americ. Annals of the Missouri Botanical Garden 80: 119-190.

Zuloaga FO, Morrone O \& Scataglini MA (2011) Monograph of Trichanthecium (Poaceae, Paniceae). Systematic Botany Monographs 94: 1-98. 